\title{
Sonochemotherapy: from bench to bedside
}

\author{
Bart H. A. Lammertink ${ }^{1 *}$, Clemens Bos ${ }^{1}$, Roel Deckers ${ }^{1}$, Gert Storm ${ }^{2,3}$, \\ Chrit T. W. Moonen ${ }^{1}$ and Jean-Michel Escoffre ${ }^{1 *}$ \\ ${ }^{1}$ Image Guided Therapy, Imaging Division, University Medical Center Utrecht, Utrecht, Netherlands, ${ }^{2}$ Department of \\ Pharmaceutical Sciences, Faculty of Science, Utrecht University, Utrecht, Netherlands, ${ }^{3}$ Targeted Therapeutics, MIRA \\ Institute for Biomedical Technology and Technical Medicine, University of Twente, Enschede, Netherlands
}

\section{OPEN ACCESS}

Edited by:

Nicolau Beckmann,

Novartis Institutes for BioMedical

Research, Switzerland

Reviewed by:

Maja Cemazar

Institute of Oncology Ljubljana,

Slovenia

Twan Lammers,

Rheinisch-Westfälische Technische Hochschule Aachen University,

Germany

${ }^{*}$ Correspondence:

Bart H. A. Lammertink and Jean-Michel Escoffre,

Image Guided Therapy, Imaging Division, University Medical Center

Utrecht, Heidelberglaan 100,

P.O. Box 85500, 3508 GA, Utrecht,

Netherlands

b.h.a.lammertink@umcutrecht.nl;

jean-michel.escoffre@univ.tours.fr

Specialty section

This article was submitted to Experimental Pharmacology and Drug

Discovery,

a section of the journal

Frontiers in Pharmacology

Received: 01 May 2015

Accepted: 22 June 2015

Published: 10 July 2015

Citation:

Lammertink BHA, Bos C, Deckers $R$ Storm G, Moonen CTW and Escoffre $J-M$ (2015) Sonochemotherapy: from bench to bedside.

Front. Pharmacol. 6:138. doi: 10.3389/fphar.2015.00138
The combination of microbubbles and ultrasound has emerged as a promising method for local drug delivery. Microbubbles can be locally activated by a targeted ultrasound beam, which can result in several bio-effects. For drug delivery, microbubble-assisted ultrasound is used to increase vascular- and plasma membrane permeability for facilitating drug extravasation and the cellular uptake of drugs in the treated region, respectively. In the case of drug-loaded microbubbles, these two mechanisms can be combined with local release of the drug following destruction of the microbubble. The use of microbubble-assisted ultrasound to deliver chemotherapeutic agents is also referred to as sonochemotherapy. In this review, the basic principles of sonochemotherapy are discussed, including aspects such as the type of (drugloaded) microbubbles used, the routes of administration used in vivo, ultrasound devices and parameters, treatment schedules and safety issues. Finally, the clinical translation of sonochemotherapy is discussed, including the first clinical study using sonochemotherapy.

Keywords: ultrasound, microbubble, sonoporation, chemotherapeutic drug, drug delivery, sonochemotherapy

\section{Introduction}

Cancer presents the second leading cause of death in the European Union with 3.45 million new cases of cancer and 1.75 million deaths from cancer in 2012 (Ferlay et al., 2013). Although a lot of progress has been made in the treatment of several cancers, many types of cancer are still lacking effective treatment options. Surgery, radiotherapy, and chemotherapy are the standard treatment possibilities and they are often combined to improve patient outcome.

While for most advanced cancers, chemotherapy remains the treatment of choice, it is rarely curative for solid tumors (Qin et al., 2015). To be successful, sufficient quantities of chemotherapeutic drugs have to reach the interior of tumor cells. Most small molecular weight chemotherapeutics $(<4 \mathrm{kDa})$ are rapidly cleared from the circulation (e.g., $\mathrm{t}_{1 / 2}<15 \mathrm{~min}$ for 5 fluorouracil, 5-FU), which is a limiting factor for drug accumulation in the tumor. In addition to challenges related to the physicochemical properties of drugs, tumors also possess physiological barriers (Jain, 2001). Contrary to healthy tissues, tumor tissues have a high interstitial fluid pressure (IFP), which is related to the lack of functional lymphatics and the leaky tumor vasculature (Boucher et al., 1990). These high pressures establish an outward fluid motion from the core of the solid tumor to the periphery and reduce fluid infiltration across the vascular wall. Thus, even if the leaky vasculature permits drug extravasation, diffusion-driven drug penetration deeper into the tumor tissue is severely restricted due to the high IFP. The increase in mean distance between 
vessels and tumor cells following tumor growth is another constraint for sufficient delivery of drugs. High tumor cell proliferation results in tumor cells forcing vessels apart, leading to a decrease in vascular density and a limitation in the access of drugs to distant tumor cells (Minchinton and Tannock, 2006). In addition, the presence of high levels of extracellular matrix limits the interstitial transport of drugs (Weinberg, 2014). Altogether these barriers oppose sufficient and uniform distribution of drugs in solid tumors, thereby limiting the therapeutic success of chemotherapy.

In addition, reaching the target site is not a guarantee that a drug will be effective. As most chemotherapeutic drugs need to enter the cell to become active, they need to pass the cell membrane. For several hydrophilic and charged drugs, e.g., bleomycin, this is a serious challenge and requires active uptake through plasma membrane transporters, which are not always present in the target cells (Pron et al., 1999).

In order to improve the efficiency of anti-cancer chemotherapeutics, physical methods including electroporation, laser, and magnetic fields have been developed (Sersa et al., 2008; Podaru et al., 2014; Sklar et al., 2014). The general principle of physical methods is based on the transient disruption of endothelial barrier and tumor cell membrane in order to facilitate the drug extravasation and the drug uptake into the endothelial and tumor cells. In recent years, research in the field of microbubble-assisted ultrasound (also known as sonoporation) aimed at delivering therapeutic molecules in vitro and in vivo has grown rapidly (Aryal et al., 2014; Azagury et al., 2014; Kiessling et al., 2014; Rychak and Klibanov, 2014; Unga and Hashida, 2014; Unger et al., 2014). Microbubble-assisted ultrasound transiently increases the permeability of biological barriers, such as blood vessel walls (i.e., drug extravasation) and cellular membranes (i.e., cellular uptake of drugs), thus enhancing the local delivery of therapeutic molecules across these barriers in the targeted region (Lentacker et al., 2014). Nowadays, the great potential of this modality for cancer therapy is clearly shown in an increasing number of publications on in vitro and in vivo drug delivery using microbubble-assisted ultrasound (Tables $\mathbf{1}$ and $\mathbf{2}$, respectively). This method is a non-invasive, easy to apply, and cost-effective treatment modality, that can be used to deliver a wide range of anticancer molecules including low molecular weight chemotherapeutic agents (sonochemotherapy), nucleic acids and monoclonal antibodies to a target site, e.g., tumor (Escoffre et al., 2013c; Ibsen et al., 2013; Unga and Hashida, 2014). In addition, this method offers the possibility to treat superficial (e.g., skin) as well as deep organs (e.g., brain, liver, prostate), under the guidance of medical imaging modalities (magnetic resonance imaging, ultrasound imaging; Kinoshita et al., 2006; Deckers and Moonen, 2010; Lammers et al., 2015).

This review first focuses on the biological effects of microbubble-assisted ultrasound (i.e., increasing plasma membrane- and vascular endothelium permeability) and subsequently on in vitro and in vivo chemotherapeutic drug delivery studies using microbubble-assisted ultrasound for cancer treatment. The limitations and future developments of sonochemotherapy will be further discussed.

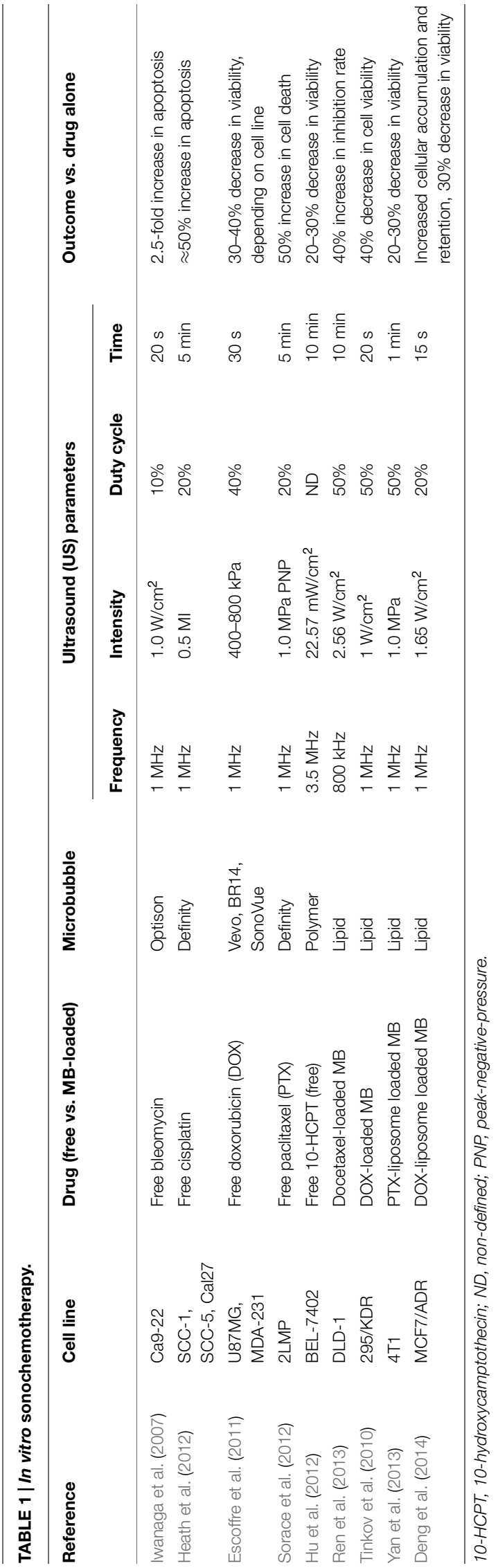




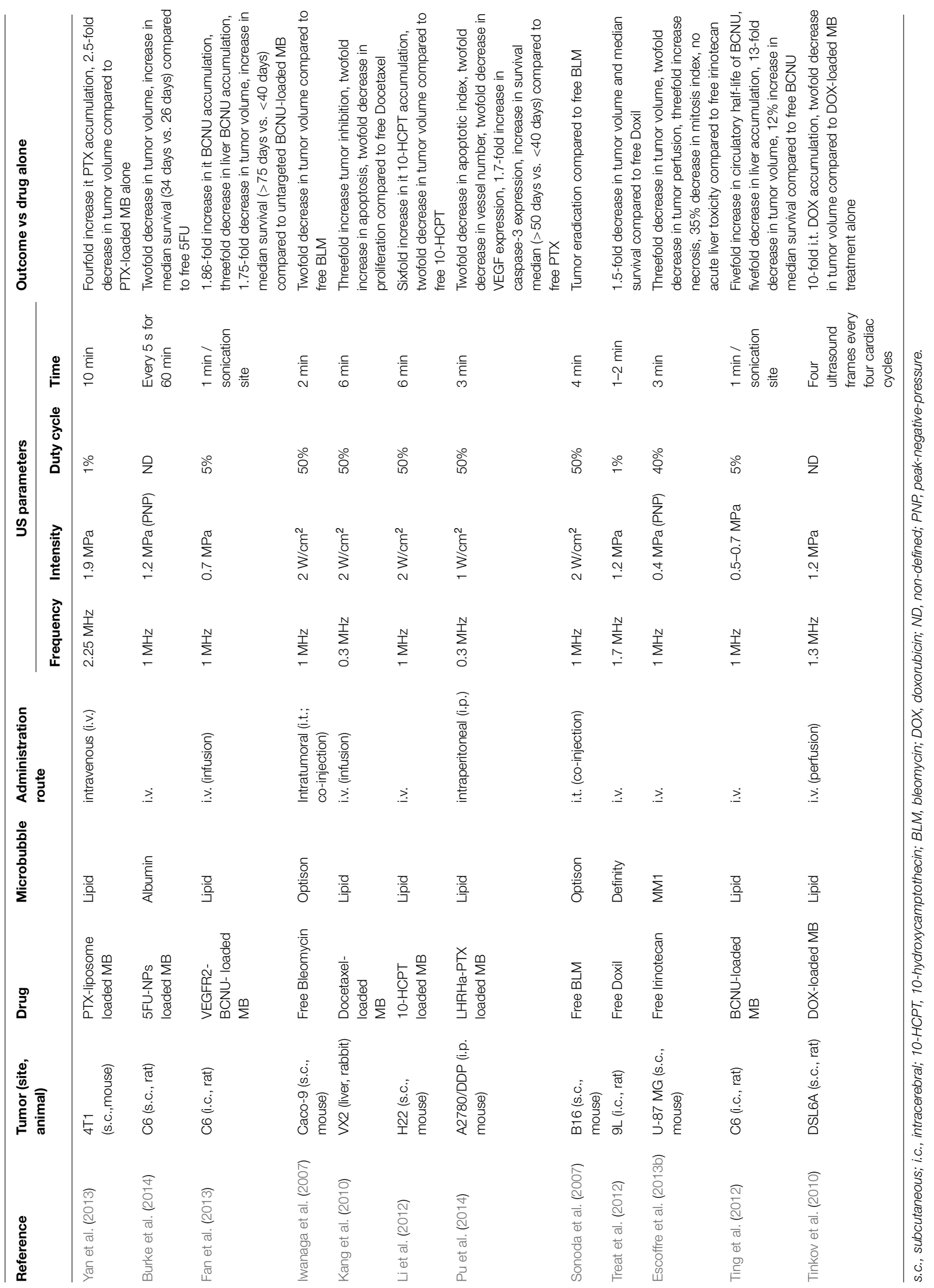




\section{Microbubble-Assisted Ultrasound}

The combination of high frequency ultrasound $(1-10 \mathrm{MHz})$ and ultrasound contrast agents (i.e., consisting of gas microbubbles) was introduced as a promising method in improving the therapeutic efficacy of drugs by increasing local delivery, while minimizing side effects to healthy tissues (Price et al., 1998). In this paper, we refer to this combination as microbubbleassisted ultrasound. The first generation of microbubbles was composed of air encapsulated by albumin $\left(\right.$ Albunex $^{\circledR}$ ) or galactose/palmitic acid (Levovist ${ }^{\circledR}$ ) shells. However, such airfilled microbubbles dissolve in the bloodstream within a few seconds after intravenous (i.v.) administration because of the high solubility of air in blood and their low resistance to arterial pressure gradients. To overcome these issues, a second generation of microbubbles was developed, which were filled with heavy-weight hydrophobic gas (e.g., perfluorocarbon, sulfur hexafluoride) encapsulated by a biocompatible shell (e.g., lipids, polymer; Hernot and Klibanov, 2008; Sirsi and Borden, 2014; Figure 1A). In studies on drug delivery by microbubble-assisted ultrasound, the bubbles are mixed with cells in vitro or injected in vivo intravascularly or directly into the tissue of interest. Microbubble behavior in an ultrasound field has been widely studied, which led to more understanding and subsequent control of the induced bio-effects that can be used for drug delivery (Kooiman et al., 2014). The response of a microbubble to ultrasound waves depends on the acoustic parameters used, such as frequency, pressure levels, and pulse duration. In short, microbubbles stably oscillate over time upon exposure to a low acoustic pressure, a process termed stable cavitation (Figures 1B and 2). These oscillations generate fluid flows surrounding the bubble, known as acoustic micro-streaming, and when in close contact with cells, result in shear stress on the cell membrane, leading to cellular uptake of drugs (Leighton, 1994; Wu, 2002; Doinikov and Bouakaz, 2010). At higher acoustic pressures, microbubbles oscillate more rigorously, leading to their violent collapse and destruction, i.e., inertial cavitation (Figure 2). Microbubble disruption can be accompanied by generation of shock waves in the medium close to the microbubbles (Junge et al., 2003; Ohl and Wolfrum, 2003). The ultrasound-induced collapse of the microbubble can be asymmetrical, leading to the formation of high velocity jets (Postema et al., 2005; Ohl et al., 2006). While shock waves induce shear stress to cells in close proximity, resulting in membrane permeability, the high velocity jets can pierce the cell membrane, and thereby create permeability. Stable and inertial cavitation are both exploited to transiently increase the permeability of biological barriers, including the vascular endothelium and plasma membrane, and therefore enhance the extravasation and the cellular uptake of drugs (Lentacker et al., 2014; Figure 2).

\section{Extravasation of Drugs}

Microbubbles are intravascular contrast agents, which do not cross the vascular endothelium (Wilson and Burns, 2010). Cavitating microbubbles close to the endothelial wall can result in several bio-effects including vascular disruption, vasoconstriction, or even shutdown of the vessels (Goertz, 2015). Several studies observed that microbubble-assisted ultrasound increased (model-) drug extravasation by stimulating paracellular (i.e., disruption of tight junctions) and transcellular pathways (i.e., transcytosis), both in vitro as well as in vivo (Figure 2;
A

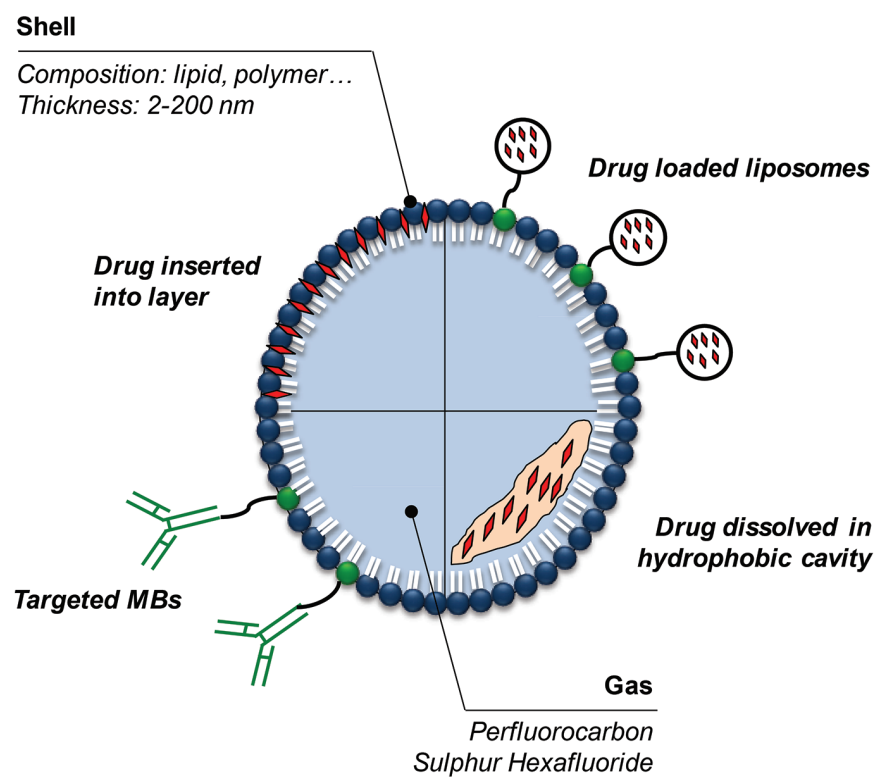

B

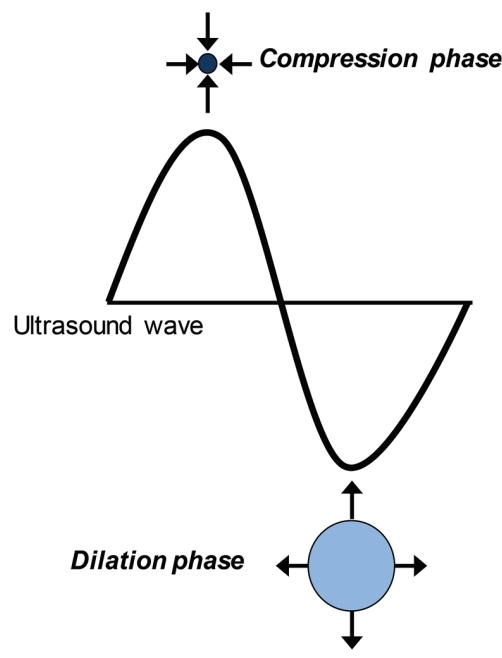

FIGURE 1 | Microbubbles and ultrasound. (A) Different options for drug-loading or targeting of microbubbles. (B) Microbubble oscillations under ultrasound exposure. 


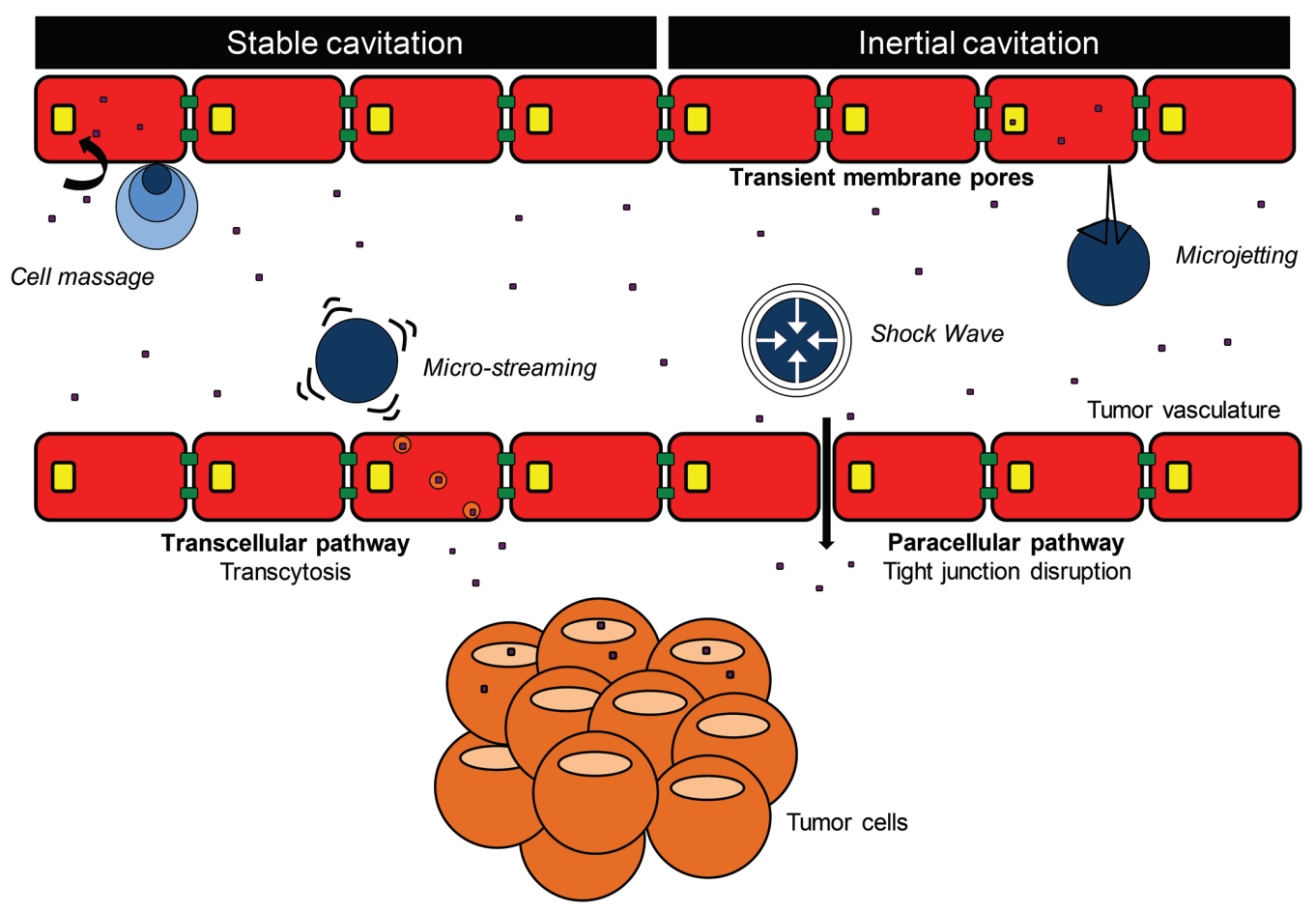

FIGURE 2 | Extravasation and cellular uptake of drug using microbubble-assisted ultrasound.

Price et al., 1998; Sheikov et al., 2008; Juffermans et al., 2009; Kooiman et al., 2010). In an in vitro endothelial barrier model, Kooiman et al. (2010) showed that microbubble-assisted ultrasound induced a $40 \%$ decrease in transendothelial electric resistance showing a loss of endothelial barrier integrity. In addition, Juffermans et al. (2009) showed that microbubbleassisted ultrasound significantly affected the integrity of in vitro endothelial monolayers by the destabilization of the tight junctions. At low acoustic pressures $(1 \mathrm{MHz}, 0.1 \mathrm{MPa})$, the integrity of the in vitro endothelial barrier was restored within $30 \mathrm{~min}$. In vivo, an acoustical pressure threshold ranging from 0.1 to $0.75 \mathrm{MPa}$ was required to enhance the extravasation of intravascular agents (e.g., red blood cells, imaging tracers, fluorescent dyes, or drugs) in skeletal muscle (Price et al., 1998), brain (Raymond et al., 2007; Sheikov et al., 2008), liver (Gao et al., 2012), and tumor (Bohmer et al., 2010; Hu et al., 2012) tissues. This extravasation occurs through tight junctions between endothelial cells $(0.2-200 \mu \mathrm{m}$; Price et al., 1998; Song et al., 2002; Stieger et al., 2007). In vivo, the integrity of the blood-brain barrier was restored within 1-4 $\mathrm{h}$ following ultrasound exposure (Sheikov et al., 2008; Ting et al., 2012). However, Marty et al. (2012) showed that the duration of extravasation after ultrasound exposure depends on the particle size. The microbubble-assisted ultrasound enhanced transcellular pathways (e.g., transcytosis) have been mainly investigated on the brain vasculature (Raymond et al., 2007; Sheikov et al., 2008; Deng et al., 2012). They reported that low (1 MHz, 0.2 MPa) and high (1.63 MHz, 1-3 MPa) acoustic pressures increased the number of transcytotic vesicles on both the luminal and abluminal surface of the endothelium. Sheikov et al. (2004) hypothesized that the transient vasoconstriction constitutes a potential cause for the increased transcytosis in vivo. In addition, Hu et al. (2012) showed that the destruction of microbubbles with a high acoustic pressure $(5 \mathrm{MHz}, 2 \mathrm{MPa})$ decreased the tumor blood flow for $30 \mathrm{~min}$ before it returned back to normal, without an increase in hemorrhage. Whereas it was demonstrated that the extravasation of fluorescent dextrans was enhanced during this period, the authors did not investigate whether transcytosis was involved. Transient vasoconstriction has been only reported in mice, which exhibit higher vasomotor excitability than other rodents and animal species.

\section{Heating and Acoustic Radiation Force}

Besides cavitation, ultrasound can also induce heating and acoustic radiation force (ARF) to improve the extravasation of drugs (Deckers and Moonen, 2010). Heating can result from the absorbance of acoustic energy as the ultrasound beam propagates through tissue. Mild heating of a tumor $\left(41-43^{\circ} \mathrm{C}\right.$ for $10-$ $60 \mathrm{~min}$ ) may improve the therapeutic efficacy of drugs by acting on tumor hemodynamics (Figure 3): (i) by increasing tumor perfusion, thus enhancing drug bioavailability in tumor tissue (Song, 1984); (ii) by increasing vascular permeability (Lefor et al., 1985; Kong et al., 2001) and reducing tumor interstitial pressure (Vaupel and Kelleher, 2012), leading to better drug penetration within tumor tissue. In addition, local heating can act as an external trigger for drug release from a carrier, e.g., thermosensitive nanoparticles (Yatvin et al., 1978; Lindner et al., 2004; Manzoor et al., 2012; Hijnen et al., 2014; Al Sabbagh 


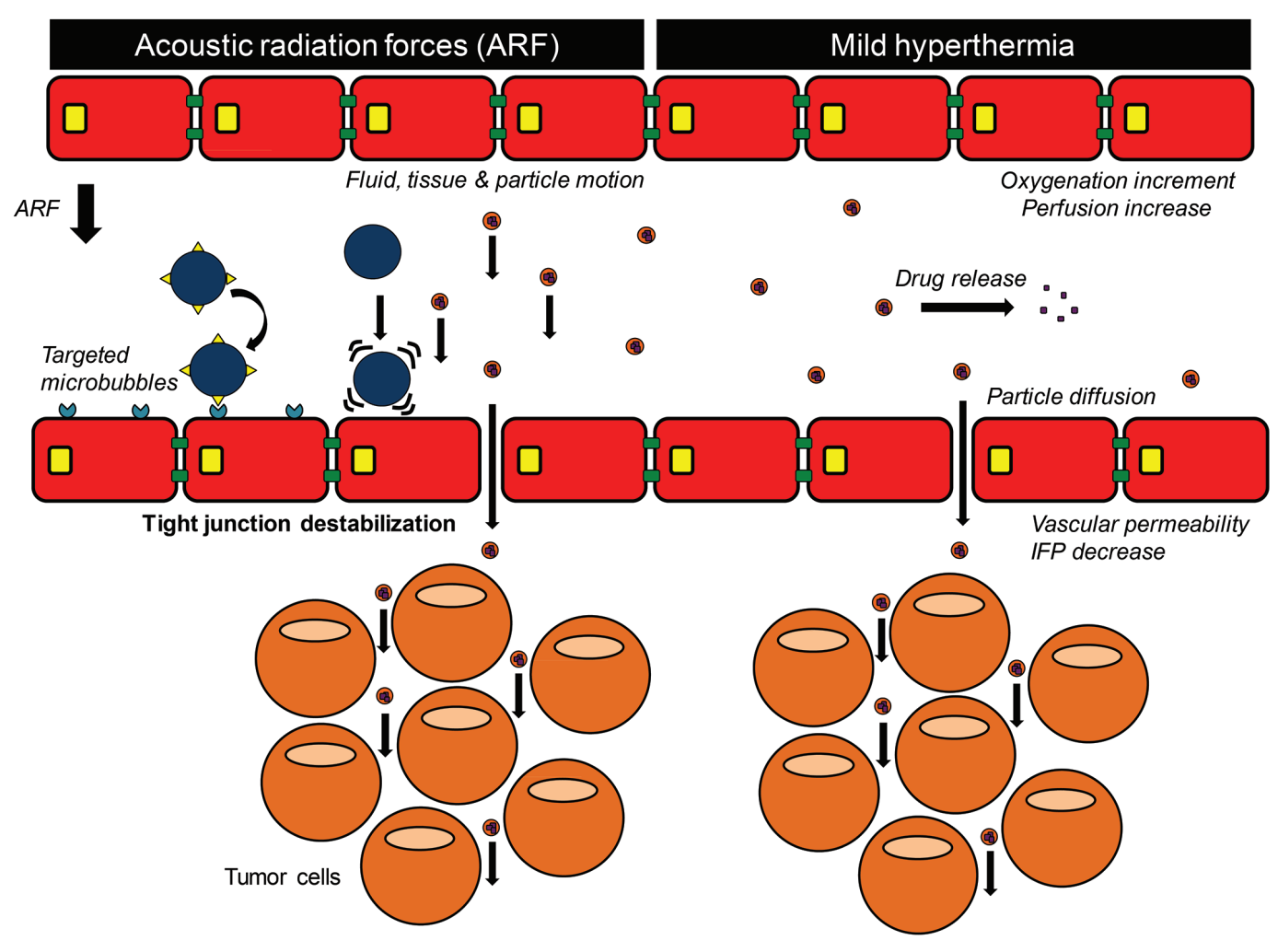

FIGURE 3 | Acoustic radiation forces and ultrasound-induced hyperthermia.

et al., 2015). Ultrasound can also generate directional ARF on molecules along its propagation path (Sarvazyan et al., 2010; Figure 3). This enhances the extravasation of free drug or drugloaded nanoparticles into tumor tissue by causing tissue shear stress and opening of endothelial tight junctions (Seidl et al., 1994; Mesiwala et al., 2002). ARF induces fluid streaming through the interstitium, thus improving biodistribution of intravascular dyes and drugs in the target tissue (Lum et al., 2006; Hancock et al., 2009). Using optical imaging, Shortencarier et al. (2004) showed that the application of ARF induced visible aggregates of fluorescent dye-loaded gas lipospheres in the direction of the beam on the far vessel wall. The lipospheres disappeared when the ARF pulses were turned off (Shortencarier et al., 2004). In addition to lipospheres, ARFs can push circulating microbubbles toward the endothelial wall, thereby improving microbubble-cell contact, which might enhance cavitationmediated extravasation of intravascular compounds (Rychak et al., 2005; Wang et al., 2014). Using ultrasound imaging, Frinking et al. (2012) reported that ARF (38 kPa PNP, 95\% DC) induced a sevenfold increase in the binding of VEGFR2-targeted microbubbles (also known as BR-55) on the endothelial wall in a prostate adenocarcinoma rat model compared with the binding without ARF.

\section{Cellular Uptake of Drugs}

Cavitating microbubbles in the vicinity of the plasma membrane can result in cell permeabilization by creating membrane pores and stimulating the endocytosis pathways, thereby facilitating intracellular drug uptake. Based on the uptake or release of non-permeant dyes (Meijering et al., 2009; Kaddur et al., 2010) and by measuring changes in membrane electrophysiology (Tran et al., 2007; Juffermans et al., 2008), previous studies showed that microbubble-assisted ultrasound induced a transient increase in membrane permeability through the generation of transient hydrophilic pores. The intracellular delivery of molecules through membrane pores is likely governed by passive diffusion or by ultrasound-mediated propulsion (i.e., microstreaming, ARF; Shortencarier et al., 2004; Lum et al., 2006). The size of these ultrasound induced pores depend on the acoustic parameters used, ranging from 1 to $94 \mathrm{~nm}$ at $0.19 \mathrm{MPa}$ PSP and from 2 to $4 \mu \mathrm{m}$ at $0.48 \mathrm{MPa}$ PSP (Yang et al., 2008).

In addition to hydrophilic pore formation, enhancement of endocytosis has also been demonstrated following microbubble-assisted ultrasound exposure (Meijering et al., 2009). Electrophysiological studies reported that microbubbleassisted ultrasound induced an influx of $\mathrm{Ca}^{2+}$, followed by an activation of $\mathrm{BK}_{\mathrm{Ca}}$ channels that results in local hyperpolarization of the cell membrane (Tran et al., 2007; Juffermans et al., 2008). At moderate ultrasound conditions ( $1 \mathrm{MHz}, 0.15-0.3 \mathrm{MPa})$, the membrane hyperpolarization facilitates the molecular uptake through endocytosis and macropinocytosis. Similar to pore formation, the contribution of endocytosis processes depends strongly on the marker size and the acoustic pressures. 
Meijering et al. (2009) reported that low acoustic pressures (1 $\mathrm{MHz}, 0.22 \mathrm{MPa} \mathrm{PNP}$ ) resulted in the cellular uptake of 4.4 and $70 \mathrm{kDa}$ fluorescent dextrans through membrane pores while the entrance of 155 and $500 \mathrm{kDa}$ fluorescent dextrans is dominated by endocytosis pathways. It should be mentioned that little is known about the faith of the agents in the endocytic vesicles, if they are degraded in the lysosome or escape from the endosome. However, De Cock et al. (2015) showed that increasing the acoustic pressures ( $1 \mathrm{MHz}, 0.5 \mathrm{MPa}, \mathrm{PNP}$ ) induced the intracellular delivery of large fluorescent dextrans (2 $\mathrm{MDa}$ ) to shift from uptake by endocytosis to uptake via the membrane pores. Regardless of the mechanism of uptake, the duration of microbubble-assisted ultrasound-mediated uptake is dependent on the plasma membrane recovery time, which is a few seconds to a few hours (van Wamel et al., 2006; Lammertink et al., 2015). The different kinetics depends on the ultrasound conditions, the model drug size and the cell physiology.

\section{Anti-Cancer Drug Delivery Protocols}

As any drug delivery technique, microbubble-assisted ultrasound treatments aim to deliver optimal quantities of chemotherapeutic drugs in targeted tumor cells and tissues. The efficiency of this delivery method depends on (i) sufficient accumulation of microbubbles and drugs near tumor cells or tissues, which is directly influenced by the properties of microbubbles, drugs (i.e., plasma circulation lifetime), and tumor (i.e., vascularization, localization), as well as administration routes (i.e., intratumoral, intravenous, intraperitoneal); (ii) the acoustic conditions including ultrasound parameters (i.e., central frequency, acoustic pressure, exposure time, etc.) and devices (i.e., home-made, commercial, medical systems); (iii) treatment schedule including the time interval between the drug and/or microbubbles administration and ultrasound treatment as well as the number of microbubble-assisted ultrasound drug delivery treatments and the time interval between them. Over the past decade, the influence of these factors on drug delivery efficiency has been investigated in order to enhance the intratumoral (i.t.) accumulation of drug, thereby increasing the treatment effect, while minimizing side effects to healthy tissues. This review shows that the drug delivery efficacy varied between the tumor models used in vivo. It is commonly known in the field that the tumor type is an important determinant for successful drug delivery. This is due to the specific properties of each tumor tissue, such as differences in tissue organization, extracellular matrix, presence of necrosis and hypoxia, cell density, and the endothelial lining of the tumor vasculature (Chauhan et al., 2011). To the best of our knowledge, no comparative study between tumor tissues with different properties has been reported using microbubble-assisted ultrasound for drug delivery. However, unlike many other drug delivery strategies, sonochemotherapy does not depend on the enhanced permeability and retention (EPR) effect, which is very heterogeneous between or within tumors, and often overestimated (Lammers et al., 2012). Interestingly, you could argue that the largest effect of sonochemotherapy can be expected in tissues with 'non-leaky' vessels, such as the brain (Ting et al., 2012), since the potential of increasing extravasation is highest. An overview of different drug delivery protocols and outcomes in vitro and in vivo are shown in Tables $\mathbf{1}$ and 2, respectively. It should be noted that this is not a complete overview, but rather a selection of different drug delivery protocols.

\section{Microbubbles}

In most studies, clinically approved microbubbles (i.e., SonoVue ${ }^{\circledR}$, Definity ${ }^{\circledR}$ ) for ultrasound imaging are employed for drug delivery. The use of these microbubbles may facilitate the clinical translation of sonochemotherapy, but any undesired side effect might have a negative impact on the use of these microbubbles in ultrasound-based diagnostics. Modification of these microbubbles (e.g., drug-loaded microbubbles) for therapeutic applications will delay clinical translation, requiring new authorization from the regulatory and health authorities.

\section{Coadministration of Microbubbles and Drug}

The simplest method for drug delivery using microbubbleassisted ultrasound is to use coadministration (Heath et al., 2012; Unga and Hashida, 2014). This approach includes drugs that are administered in patients anyway in current clinical practice, with the addition of an injection of (clinically approved) microbubbles. Microbubbles and drugs can be mixed in solution in vitro and the mixture is then injected in vivo. This strategy offers two main advantages: (i) both constituents can be handled completely separately until in vitro or in vivo administration; (ii) instead of mixing microbubbles and drug before injection, two separate injections of the constituents can also be performed, thus allowing drugs to reach plasma peak levels before injecting microbubbles (Escoffre et al., 2013b). Microbubbles have a short circulation time and therefore need to be exposed to ultrasound within minutes after injection, otherwise they will be degraded and unable to induce bioeffects. The coadministration approach seems to be the best strategy for in vitro purposes (Escoffre et al., 2011; Sorace et al., 2012) or, in vivo, i.t. injection of the mixture (Sasaki et al., 2014), where similar spatio-temporal distribution of both components will be ensured. Iwanaga et al. (2007) showed that the in vitro delivery of bleomycin using microbubbleassisted ultrasound induced twofold decrease in cell viability compared to the bleomycin treatment alone (Table 1). In vivo, they reported that the exposure of a tumor to ultrasound following the i.t. co-injection of microbubbles and bleomycin also resulted in a twofold decrease in tumor volume (Iwanaga et al., 2007). Kotopoulis et al. (2014) coadministered commercially available microbubbles and gemcitabine i.v. in a pancreatic cancer model in mice. They showed that ultrasound exposure (1 MHz, 0.2 MPa PNP) decreased the tumor volume twofold compared to gemcitabine alone (Kotopoulis et al., 2014). Opposed to the advantages of coadministration using clinically approved microbubbles and drugs that allow clinical translation, there are also disadvantages. The main limitations of the i.v. 
injection of microbubble/drug mixture compared to drug-loaded microbubbles are: (i) differential distribution of both constituents because of their physicochemical properties; (ii) fast degradation of free drugs and microbubbles; (iii) unspecific accumulation of free drugs in the healthy tissues.

\section{Drug-Loaded Microbubbles}

To overcome these limitations of i.v. coadministration, microbubbles have been modified to function not only as cavitation nuclei, but also as drug delivery carriers. For example, lipophilic drugs can be incorporated into the lipid monolayer shell of microbubbles or dissolved in an oil pocket between the gas core and the microbubble's shell (Ibsen et al., 2013). By applying this approach, Burke et al. (2014) found that the application of ultrasound ( $1 \mathrm{MHz}, 1.2 \mathrm{MPa}$, every $5 \mathrm{~s}$ for $60 \mathrm{~min}$ ) on subcutaneous C6 glioma tumor following the i.v. injection of 5-FU-loaded microbubbles $\left(1 \times 10^{5}\right.$ microbubbles/g body weight) led to twofold decrease in tumor volume compared to 5-FU treatment alone (Burke et al., 2014). While these approaches seem to be promising, the low drug loading capacity of microbubbles is a major drawback. Consequently, the use of drug-loaded microbubbles requires either enhancement of the drug loading efficiency, administration of high dose of drug-loaded microbubbles, or application of consecutive treatments.

The small size of microbubbles and their gaseous lumen restricts the space for drug loading. Recent publications reported that the binding of drug-loaded nanoparticles on the microbubble's surface could increase the amount of loaded drug (Geers et al., 2011). The loading efficiency can be further improved by applying multiple layers of drug-loaded nanoparticles around the microbubble shell. The binding of drugloaded nanoparticles on microbubbles may not be necessary for polymer-based microbubbles, as significant amounts of (model) drug can be loaded into the polymer-based shell (Fokong et al., 2012). Cochran et al. (2011) showed that the loading capacity is higher for hydrophobic drugs compared to hydrophilic drugs, and that the acoustic properties of the microbubbles were unaffected (Cochran et al., 2011).

Based on current studies, a high dose of drug-loaded microbubbles, i.e., $>10^{10}$ microbubbles, must be intravenously injected to reach a therapeutic dose similar to the one used in clinical chemotherapy. However, the recommended diagnostic doses of microbubbles currently approved for contrast-enhanced ultrasound imaging (e.g., SonoVue ${ }^{\circledR}$, Definity ${ }^{\circledR}$ ) are between $10^{9}$ and $10^{10}$ microbubbles for an $80-\mathrm{kg}$ adult (Wilson and Burns, 2010). Nevertheless, preclinical and clinical studies have reported a good tolerance with 100- and 1000-fold higher doses of these microbubbles in non-human primates and patients (Grauer et al., 1996; Bokor et al., 2001). Consequently, the injection of a high dose of drug-loaded microbubbles may not be a limitation for clinical use, but further preclinical studies might be necessary to identify any potential toxicity of high concentrations of liposome and shell's components (i.e., lipid, polymer, and albumin).

Finally, several preclinical studies reported the use of repeated sonochemotherapy treatments (Kang et al., 2010; Tinkov et al.,
2010; Li et al., 2012; Ting et al., 2012). For example, Li et al. (2012) reported that the repetitive treatment (i.e., once a day for seven consecutive days) of subcutaneous hepatic tumor using 10hydroxycamptothecin-loaded microbubbles $(4 \mathrm{mg} / \mathrm{kg}$ ) induced twofold stronger decrease in tumor volume in a subcutaneous hepatic tumor model $\left(1 \mathrm{MHz}, 2 \mathrm{~W} / \mathrm{cm}^{2}, 6 \mathrm{~min}\right)$ compared to the 10-hydroxycamptothecin-based chemotherapy alone (Li et al., 2012).

\section{Targeted Microbubbles}

Microbubbles can be modified to target specific overexpressed markers on tumor cells (i.e., PSMA, prostate specific membrane antigen; LHR, luteinizing hormone receptor) or tumor microvasculature (VEGF-R2, vascular endothelial growth factor receptor -2) through attachment of targeting ligands or antibodies onto the microbubble's shell (Kiessling et al., 2012, 2014; Novell et al., 2013). This may lead to enhanced accumulation of the microbubbles in the target tumor cells or tissues. For example, Fan et al. (2013) designed targeted BCNU-loaded microbubbles, which bind the VEGF-R2 overexpressed on tumor microvasculature (VEGFR2-BCNUloaded microbubbles; Figure 4A). The exposure of orthotopic glioma to ultrasound $(1 \mathrm{MHz}, 0.7 \mathrm{MPa}, 1 \mathrm{~min} /$ sonication site) following i.v. injection of VEGFR2-BCNU-loaded microbubbles (1.25 mg BCNU) resulted in 1.75-fold decrease in tumor volume compared to the untargeted BCNU-loaded microbubbles (Figure 4B; Fan et al., 2013). The use of microbubbles targeting overexpressed markers on the tumor cells themselves is limited to in vitro drug delivery, i.t. or intraperitoneal (i.p.) injection of microbubbles and drugs, primarily because the microbubbles, when administrated intravenously, cannot extravasate due to the size (Cavalieri et al., 2010). For imaging, several groups have reported on the in vivo accumulation of targeted microbubbles in the tumor microvasculature by binding inflammation markers overexpressed on tumor endothelial cells (Deshpande et al., 2010). Although these microbubbles were designed as ultrasound contrast agents for molecular imaging, it might be possible to develop optimal tissue- or organ-selective drug delivery agents by combining targeting capacities and drug loading of microbubbles (Kiessling et al., 2012). However, no evidence of their use for drug delivery has been reported yet.

To summarize, the coadministration of drugs/microbubbles and drug-loaded microbubbles can both be used for drug delivery. The coadministration approach is likely to be the fastest way into the clinic, as it combines clinically approved drugs and microbubbles. However, the drugloaded microbubbles may hold the greatest therapeutic potential, as it locally releases the drug upon ultrasound exposure. Since this approach represents new therapeutic entities, such 'therapeutic microbubbles' require extensive testing for safety and efficacy before they can be approved for clinical use. To the best of our knowledge, no study has been published that directly compares drug-loaded microbubbles with coadministration of free drugs and microbubbles at equal dosing schemes. 


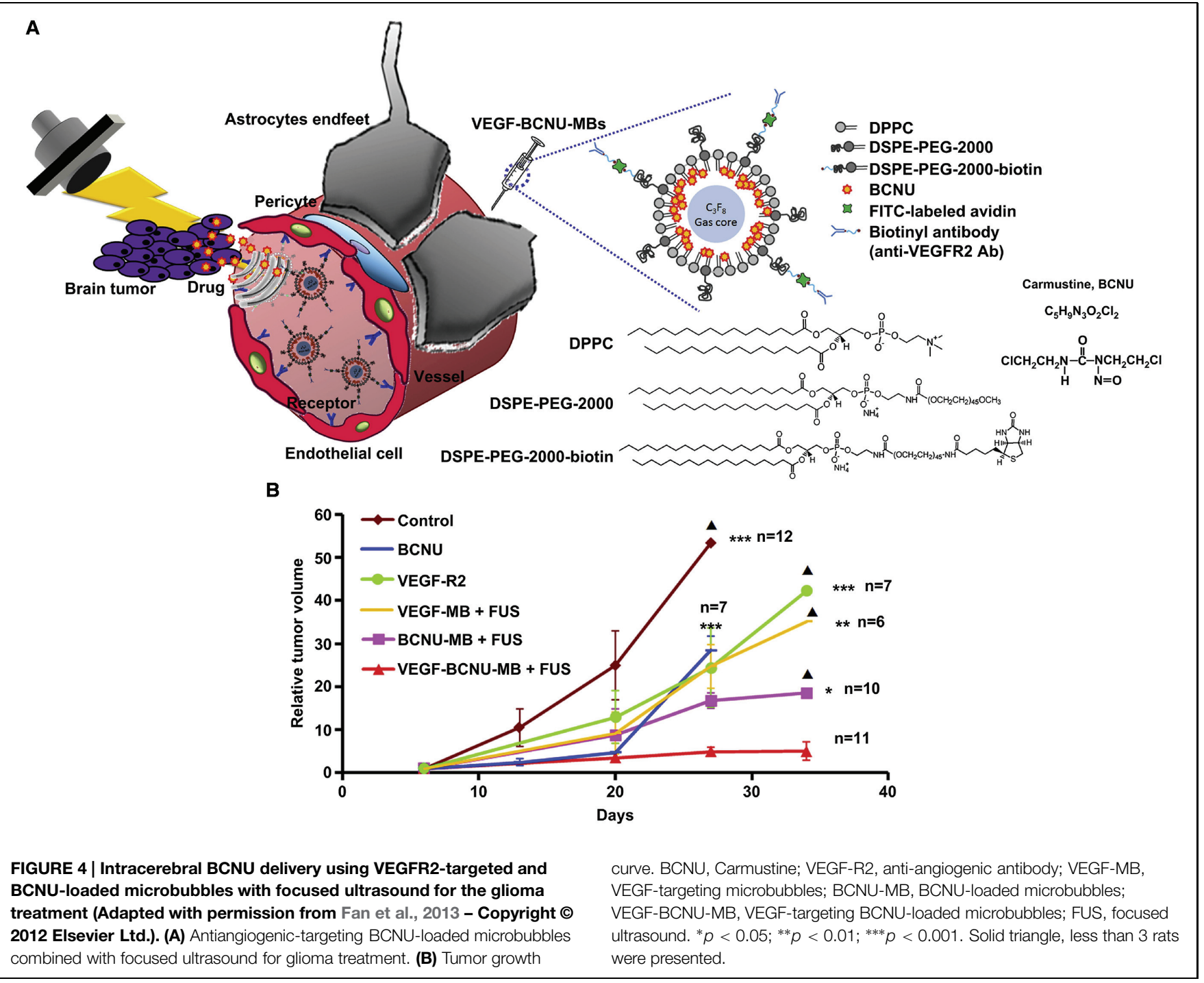

\section{Administration Routes}

The most direct administration route for drug delivery is i.t. injection (Sonoda et al., 2007; Sasaki et al., 2014). The advantages of i.t. administration over systemic injection include the circumvention of the transvascular barrier and the generation of transient interstitial pressure gradients. The latter can induce convection and tissue deformation, which can decrease the connectedness of the extracellular matrix and size of pores in the tumor interstitial space (Frenkel, 2008). By using i.t. administration, a high drug dose can be directly delivered into the target tumor while minimizing its side effects toward healthy tissues. This administration route overcomes the drawback related to the short plasma half-life of drugs and microbubbles after i.v. injection. In addition, this route is most interesting for hydrophilic small chemotherapeutic drugs that have difficulties to enter tumor cells. By applying i.t. injection, microbubbles and drugs are distributed within the tumor by diffusion and convection, and subsequent US exposure will result in drug uptake in tumor cells. However, in i.t injection, there are some limitations such as the injected volume and the accessibility of the tumor site, which restrict the application of microbubbleassisted ultrasound to superficial tumors such as melanoma, and cutaneous and subcutaneous tumors.

For deep-seated tumors, most protocols recommend injection of drugs and microbubbles via blood flow, providing better access to deeper tumors (Treat et al., 2012; Yan et al., 2013; Burke et al., 2014). The i.v. route is a relatively easy and safe way to be used in the clinic for the administration of therapeutics and microbubbles. As previously described, the main limitation of this administration route is the rapid clearance of drug from plasma and the unspecific accumulation of this drug in healthy tissues. Therefore, drugs can be loaded on microbubbles to overcome these shortcomings (Ting et al., 2012; Sirsi and Borden, 2014). The success of i.v. drug delivery relies on sufficient tumor vascularization, thus restricting the application of this administration route to hypervascularized tumors. Next to extravasation, microbubble-assisted ultrasound can also increase the penetration of drugs into the tissue. 
In addition, it can "homogenize" drug uptake, since drug distribution tends to be very heterogeneous throughout the tumor. Since microbubble-assisted ultrasound will mostly affect the vascular endothelium, the i.v. route is most suitable for drugs that can benefit from ultrasound-induced extravasation and penetration or intracellular delivery in endothelial cells.

Recent studies reported that the i.p. injection may be useful for drug delivery using microbubble-assisted ultrasound for the treatments of primary peritoneal cancers or cancers with i.p. metastases. $\mathrm{Pu}$ et al. (2014) investigated the i.p. delivery of paclitaxel (PTX) for the treatment of ovarian cancer using luteinizing hormone-releasing hormone analog (LHRHa) targeted and PTX-loaded microbubbles (20 mg/kg PTX) and ultrasound $\left(0.3 \mathrm{MHz}, 1 \mathrm{~W} / \mathrm{cm}^{2}, 3 \mathrm{~min}\right)$. This therapeutic protocol led to a twofold increase in apoptotic index and a 2.5 -fold decrease in vessel number compared to the single injection of free PTX or PTX delivery using ultrasound alone (Pu et al., 2014). Due to the microbubble size, penetration of the microbubbles by convection throughout the tumor is hindered, thereby limiting the tumor cell binding to the peripheral rim of the tumor. Nevertheless, the targeted microbubbles in this study showed superior efficacy compared to the untargeted bubbles.

\section{Ultrasound Devices, Transducer, and Parameters}

Several investigations showed extensive optimization of the acoustic parameters to result in an efficient and safe in vitro and in vivo drug delivery. Among these studies, clinical ultrasound scanners have been used to deliver drugs using microbubbleassisted ultrasound (Tinkov et al., 2010; Sasaki et al., 2014), which has the advantage of enabling both imaging of- and drug delivery to the targeted tumor. However, the ultrasound settings that are allowed on such equipment are limited for safety reasons. Specific ultrasound parameters [low cycles and mechanical index (MI) $0.5<\mathrm{MI}<1.9$ ] are used to destroy microbubbles during a diagnostic tissue perfusion study (Szabo, 2013). However, such parameters might not be efficient for drug delivery. In addition, clinical ultrasound probes are unfocused and thus the ultrasound energy will have substantial effects in the regions surrounding the target tissue. Clinical ultrasound scanners are "black-boxes" which do not allow controlling all ultrasound parameters. Hence, home-made and commercial therapeutic ultrasound devices have been designed to control many ultrasound parameters, which can subsequently be optimized for drug delivery (Zhao et al., 2011; Lin et al., 2012; Escoffre et al., 2013a). Ultrasound transducers used in the literature can be focused or unfocused (Sanches et al., 2011). Focused beams are created using spherically curved transducers, which greatly increase the ultrasound intensity in a small region of interest, e.g., a tumor. Due to a lack of standardized calibration methods concerning the applied ultrasound parameters and the heterogeneity in equipment used, it is not straightforward to compare the results of most studies directly (ter Haar et al., 2011).

The transmission center frequency used for in vivo drug delivery studies listed in Table 2 ranges from 0.3 to $2.25 \mathrm{MHz}$. The choice of frequency to be used can depend on the microbubble's size and its resonance frequency, but also on the depth of the tissue to be reached, as higher frequencies suffer from increased attenuation. The resonance frequency of microbubble decreases as their size increases (Minnaert, 1933). When using a low frequency range, the acoustic pressure threshold to initiate microbubble cavitation can be reduced, thereby limiting putative tissue damage. In most of the reported investigations, $1 \mathrm{MHz}$ was used as a frequency to achieve drug delivery using microbubbleassisted ultrasound (Tables $\mathbf{1}$ and 2 ).

The ultrasound dose is usually expressed in different units depending on whether a medical ultrasound scanner, commercial or laboratory-made device is used for drug delivery (Table 2). With home-made or commercial therapeutic ultrasound devices, ultrasound exposure is usually expressed either in acoustic pressure amplitude $(\mathrm{kPa})$ or in intensity $\left(\mathrm{W} / \mathrm{cm}^{2}\right)$ while for medical ultrasound scanners, the dose is usually expressed in the terms of MI (expressed as the ratio of the peak negative pressure in $\mathrm{MPa}$ to the square root of the frequency in $\mathrm{MHz}$ ). Among the published studies, it is not clearly stated whether ultrasound intensity are spatial averaged, temporal averaged intensity (ISATA) or spatial peak, temporal averaged intensity (ISPTA). ISATA is frequently used when non-focused transducer is employed for drug delivery. Ultrasound intensities ranging from 0.064 to $3 \mathrm{~W} / \mathrm{cm}^{2}$ (n.b., ISPTA $0.0003-0.9 \mathrm{~W} / \mathrm{cm}^{2}$ for ultrasound-based diagnostics) have been applied in recent studies to deliver drugs in tumor tissue without injuries (Kang et al., 2010; Lu et al., 2011). The MI used for in vivo drug delivery ranges from 0.2 to 2 (n.b., MI threshold for clinical diagnosis is 1.9). Drug delivery requires a minimum MI known as the permeabilization threshold, which is typically lower than 1 (Choi et al., 2007). Exposure of tumor tissues above, but near the cavitation threshold has so far yielded the most promising results of drug delivery without significant side effects. Increasing the ultrasound dose further enhanced drug delivery in the target tissue but was also accompanied by hemorrhage and tissue injuries (Kang et al., 2010; Lu et al., 2011).

The duty cycle is the percentage of time that an ultrasound device is transmitting acoustic waves. The duty cycle ranges from 0.25 to $50 \%$ for drug delivery into tumors (Table 2). To prevent thermal tissue damage, low duty cycles are used when high ultrasound intensities are applied and vice versa (Lin et al., 2012; Wei et al., 2013).

Ultrasound exposure time plays a major role in drug delivery using microbubble-assisted ultrasound. During this time, ultrasound pulses are emitted repeatedly at a pulsing interval to induce the complete destruction of microbubbles in the targeted tumor. Ultrasound exposure times from $2 \mathrm{~s}$ to 10 min have been reported (Table 2). However, exposure times of 1-5 min are recommended to prevent tissue injuries (e.g., hemorrhages; Mei et al., 2009; Yan et al., 2013).

\section{Treatment Schedule}

The therapeutic protocol depends on the duration of microbubble-assisted ultrasound-mediated permeability of tumor tissues and the pharmacokinetics of chemotherapeutic drugs. Some studies reported drug administration at different time points following the exposure of tumor to microbubbleassisted ultrasound to assess the duration of enhanced permeability (few seconds - few hours, depending on the particle 
size; Marty et al., 2012; Tzu-Yin et al., 2014; Lammertink et al., 2015). Other investigations recommend waiting for the peak concentration of drug in the blood before the administration of microbubbles and the subsequent exposure of tumors to ultrasound. For example, Escoffre et al. (2013b) succeeded to optimize therapeutic efficacy of irinotecan using microbubbleassisted ultrasound in subcutaneous glioblastoma. In this study, the protocol consisted of an i.v. injection of irinotecan followed $1 \mathrm{~h}$ later by an i.v. administration of microbubbles (Escoffre et al., $2013 \mathrm{~b})$. This delay is required to reach the maximal systemic concentration of SN-38, the active metabolite of irinotecan, in the blood. This strategy induced a twofold decrease in tumor volume and perfusion compared to irinotecan without subsequent ultrasound exposure.

In most therapeutic protocols using the coadministration approach or drug-loaded microbubbles, ultrasound was applied to the tumors immediately (5-10 s) after microbubble injection (Sonoda et al., 2007; Matsuo et al., 2011). This strategy supposes that drugs and microbubbles are sufficiently accumulated in the target tissue during the few seconds following their administration. However, no real evidence has been reported whether this is actually the case. In addition, monitoring of microbubble arrival at the target tissue using contrast-enhanced ultrasound prior to ultrasound therapy is rarely performed. At present, all investigations show that at least several consecutive treatments (2-20 times) at optimal time intervals (1 day - 1 week) are required to achieve significant decrease in tumor growth or even tumor eradication (Table 2).

\section{Therapeutic Efficacy vs. Safety: from In Vitro to Preclinical Studies}

As described above, the therapeutic benefit of drug delivery using microbubble-assisted ultrasound relies on enhancing accumulation of drugs in tumor cells or tissues and on decreasing their deposition in healthy tissues, thus reducing their side effects (Tinkov et al., 2010; Li et al., 2012; Fan et al., 2013; Burke et al., 2014). Using the coadministration approach or drug-loaded microbubbles, microbubble-assisted ultrasound enhances in vitro the therapeutic efficacy of clinically approved chemotherapeutics including doxorubicin (Dox), cisplatin, bleomycin, PTX, and docetaxel (Table 1). Most in vitro studies only monitor drug effectiveness with or without microbubble-assisted ultrasound. However, some studies also investigated the underlying mechanism. For example, Deng et al. (2014) showed enhanced intracellular Dox levels (Figure 5A) and increased retention due to a down-regulation of P-glycoprotein following ultrasound exposure in the presence of Dox-liposome loaded microbubbles. This resulted in a significant increase of double-stranded DNA breaks and reduced cell viability (Figure 5B). The exposure of tumor cells to microbubble-assisted ultrasound without any drugs had no or few effects on cell viability ( $>85 \%$ cell viability).

In in vivo studies it was clearly observed that microbubbleassisted ultrasound improves the therapeutic efficacy of drugs for different tumor animal models. However, most studies only monitor outcomes like survival and tumor size. Unfortunately, i.t. drug accumulation and distribution is often not investigated. Regardless of the administration route, only $40 \%$ of preclinical studies showed that an enhanced therapeutic efficacy could be attributed to increased i.t. drug levels. For example, Tinkov et al. (2010) demonstrated that the exposure of pancreas carcinoma in rats to ultrasound $(1.3 \mathrm{MHz}, 1.2 \mathrm{MPa} \mathrm{PNP}$, four frames of ultrasound every four cardiac cycles) after i.v. injection of DOX-loaded microbubbles (140 $\mu \mathrm{g}-3.14 \times 10^{9}$ microbubbles) induced a 10-fold increase in i.t. DOX accumulation compared to DOX-loaded microbubble injection alone (Tinkov et al., 2010). This therapeutic protocol led to a twofold decrease in tumor volume.

Next to increased drug concentration in the target tissue, one of the expected consequences of i.t. drug delivery using microbubble-assisted ultrasound is the reduction of drug deposition in healthy tissues. However, this effect is expected to be only significant for local release from drug-loaded microbubbles compared to the coadministration approach,
A

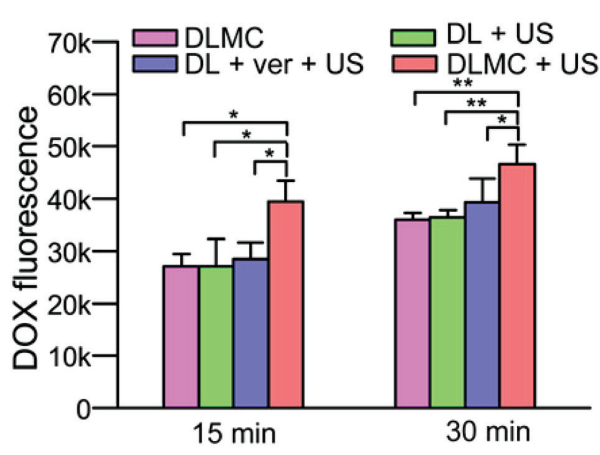

B

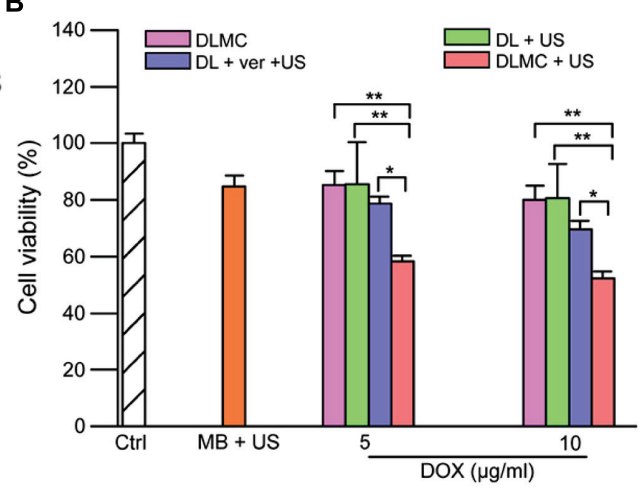

FIGURE 5 | (A) Intracellular doxorubicin (DOX) concentration in MCF-7/ADR cells 15 and 30 min post treatment. (B) Cell cytotoxicity after several treatments with or without US. DLMC, DOX-liposome-microbubble complexes; DL, DOX-liposomes; ver, verapamil; US, Ultrasound. ${ }^{*} p<0.05$, ${ }^{* *} p<0.01$ (Adapted with permission from Deng et al., 2014 - Copyright @ 2014 Elsevier Ltd.). 
where free drugs can enter healthy tissue anyway, without ultrasound exposure. Less than $10 \%$ of preclinical studies reported on drug distribution toward healthy tissues. Among the studies that do measure this, Yan et al. (2013) reported that the application of ultrasound (2.25 MHz, 1.9 $\mathrm{MPa}$, $10 \mathrm{~min}$, three treatments: one treatment every 3 days) on subcutaneous breast tumor following the i.v. injection of PTXloaded microbubbles $\left(120 \mu \mathrm{g}-1 \times 10^{9}\right.$ microbubbles $)$ resulted in fourfold increase in i.t. accumulation of PTX (Figure 6A) and 2.5-fold decrease in tumor volume compared to PTXloaded microbubbles treatment alone (Figure 6B). The authors also investigated the drug biodistribution in healthy organs including heart, liver, spleen, lung, and kidney $1 \mathrm{~h}$ after i.v. administration of the PTX-loaded microbubbles and ultrasound exposure (Yan et al., 2013). The PTX biodistribution in heart, spleen, and lung was not significantly different between mice that received PTX-loaded microbubbles treatment alone or combined with ultrasound (Figure 6A). However, the PTX delivery using microbubble-assisted ultrasound led to a slight but significant decrease in PTX concentration in liver and kidney compared to PTX-loaded microbubbles injection alone (Figure 6A). No significant loss of body weight and other adverse effects were observed during the therapeutic procedure. Moreover, Ting et al. (2012) designed a therapeutic protocol based on BCNU-loaded microbubbles $\left(0.8 \mathrm{mg}-1 \times 10^{10}\right)$ with focused ultrasound $(1 \mathrm{MHz}, 0.5-0.7 \mathrm{MPa}, 2$ sonications, $1 \mathrm{~min} /$ sonication) to improve BCNU-based chemotherapy for glioblastoma treatment. They showed that the encapsulation of BCNU in microbubbles prolonged its circulatory half-life fivefold and intrahepatic accumulation of BCNU was reduced fivefold due to the slow reticuloendothelial system uptake of BCNUloaded microbubbles (Ting et al., 2012). These microbubbles alone or in combination with focused ultrasound were associated with lower levels of aspartate- and alanine-aminotransferases compared to free BCNU, suggesting that these microbubbles may effectively reduce liver toxicity and damage. In glioblastomabearing rats, BCNU-loaded microbubbles with ultrasound led to 13 -fold decrease in tumor volume. However, median survival was extended by only $12 \%$ compared to BCNU and control.

However, for all microbubble-based ultrasound therapies, the effect on the vasculature should be closely monitored. There is a 'fine line' between stimulating vascular permeability and inducing vascular damage, which can result in inhibition of tumor perfusion. Although this may be a desired effect in some studies, for drug delivery from the vasculature, a reduced tumor perfusion might limit the i.t. drug supply. For example, Burke et al. (2011) demonstrated that the mechanical effect of low duty cycle ultrasound ( $1 \mathrm{MHz}, 1 \mathrm{MPa} \mathrm{PNP})$ in combination with microbubbles could inhibit glioma growth by blocking tumor perfusion. The anti-vascular action of microbubbleassisted ultrasound (1 MHz, 1.6 MPa PNP) was also adopted by Todorova et al. (2013) who subsequently injected an antiangiogenic agent to prevent the formation of new vessels. In the light of these results, animal studies conducted with ultrasound pressures $>1.0 \mathrm{MPa}$ should always include a control group with microbubble-assisted ultrasound only, and preferably monitor the perfusion of the exposed tissue (e.g., by Doppler or contrastenhanced ultrasound imaging).

To summarize, a growing number of preclinical investigations show promising results for future clinical applications. Future studies will have to confirm that the increase in therapeutic efficacy of sonochemotherapy is correlated with enhanced i.t. accumulation and penetration of drugs. To demonstrate the safety of this method, drug biodistribution toward healthy organs and tissues should be monitored and physiological functions of healthy organs should be examined using imaging, histological analysis, and blood biochemistry analysis. Information on in vivo biodistribution and pharmacokinetics of intact and destroyed
A

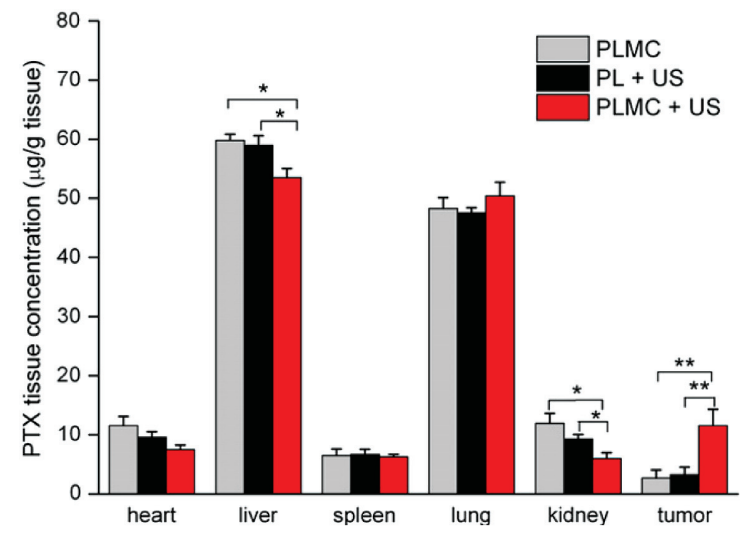

FIGURE 6 | Paclitaxel (PTX) delivery by PTX-loaded microbubble with ultrasound for breast cancer treatment (Adapted with permission from Yan et al., 2013 - Copyright $\odot 2013$ Elsevier Ltd.). (A) Paclitaxel in vivo distribution in heart, liver, spleen, lung, kidney and tumors $1 \mathrm{~h}$ after injection of paclitaxel-loaded microbubble complexes (PLMC) alone, paclitaxel liposomes
B

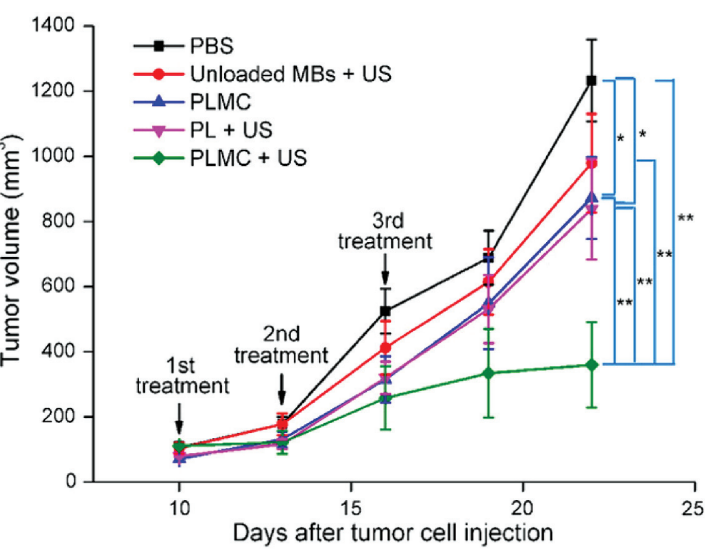

$(\mathrm{PL})+$ US or PLMC + US; (B) In vivo growth inhibition in 4T1-tumor bearing mice within 22 days. Mice were treated with PBS (squares), unloaded microbubbles + US (circles), PLMC without US (upward triangles), PL + US (downward triangles) or PLMC + US (diamonds) on days 10, 13 and 16 after tumor cell injection. Results represent mean $\pm \mathrm{SD}, n=6 .{ }^{*} p<0.05$; ${ }^{* *} p<0.01$. 
microbubbles as well as an evaluation of their systemic side effects are still absent in most available publications. These aspects need to be integrated in future studies. It must be noted that the sonochemotherapy approach has mainly been evaluated in small animals. Studies in large animals are still lacking and might face challenging and unexpected physical (e.g., ultrasound penetration depth, ultrasound attenuation) and biological (e.g., plasma life time of drug and microbubbles) limitations.

\section{Translation to the Clinics}

Despite the novelty of the field of ultrasound-mediated drug delivery, a first clinical case study has been conducted in five patients with locally advanced pancreatic cancer (Kotopoulis et al., 2013, 2015). In this study, gemcitabine was administrated by i.v. infusion at a dose of $1000 \mathrm{mg} / \mathrm{m}^{2}$ over $30 \mathrm{~min}$ (Figure 7A). During the last $10 \mathrm{~min}$ of chemotherapy, ultrasound imaging was performed in standard abdominal imaging mode to locate the position of the tumor (Figure 7B). At the end of gemcitabine infusion, when drug plasma level peaked, $0.5 \mathrm{~mL}$ of clinically approved SonoVue ${ }^{\circledR}$ contrast agents followed by $5 \mathrm{~mL}$ saline were intravenously injected every $3.5 \mathrm{~min}$ to ensure their presence throughout the whole treatment. Tumors were exposed to ultrasound (1.9 MHz, $0.49 \mathrm{MI}, 1 \% \mathrm{DC})$ using an ultrasound diagnostic scanner. The cumulative ultrasound exposure was only 18.9 s (Figure 7A). All five patients tolerated an increased number of treatment cycles compared to gemcitabine treatment without ultrasound (16 \pm 7 vs. $9 \pm 6$ cycles), reflecting an improved physical state as well as an increased survival. In two out of five patients, the maximum tumor diameter was either transiently or permanently reduced, while the other patients exhibited reduced tumor growth compared to a historical control group of 80 patients (Figure 7C; Kotopoulis et al., 2013). Compared to this historical data, survival increased with $60 \%$ (Kotopoulis et al., 2015). The authors did not report side effects related to this therapeutic protocol. Nevertheless, the true clinical benefit was not clearly established because of the low number of patients studied. The therapeutic protocol (i.e., ultrasound parameters, doses of drug, type and concentrations of microbubbles) should be optimized and long-term safety aspects have to be addressed in future investigations in a larger number of patients.

Moreover, we are referring to a safety study of combining ultrasound microbubbles and chemotherapy to treat liver metastases from gastrointestinal tumors and pancreatic carcinoma conducted by the Profs. K. Yan and L. Shen at Beijing Cancer Hospital (Yan and Shen, 2014). This study is currently recruiting patients. In this clinical trial, gemcitabine will be intravenously injected to patients with pancreatic carcinoma while oxaliplatin and taxol based chemotherapy will be administrated by i.v. perfusion to patients with liver metastases. Thirty min after chemotherapy, $1 \mathrm{~mL}$ of SonoVue ${ }^{\circledR}$ contrast agents will be intravenously injected during six times

A

$\square$ Gemcitabine infusion

$\square$ Diagnostic scanning

Ultrasound and microbubble treatment

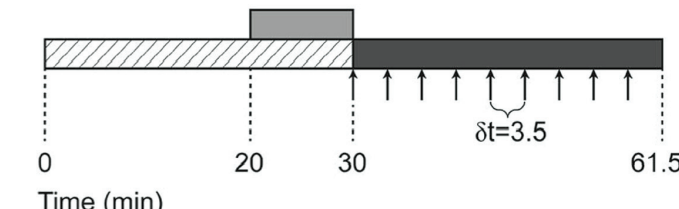

C

B
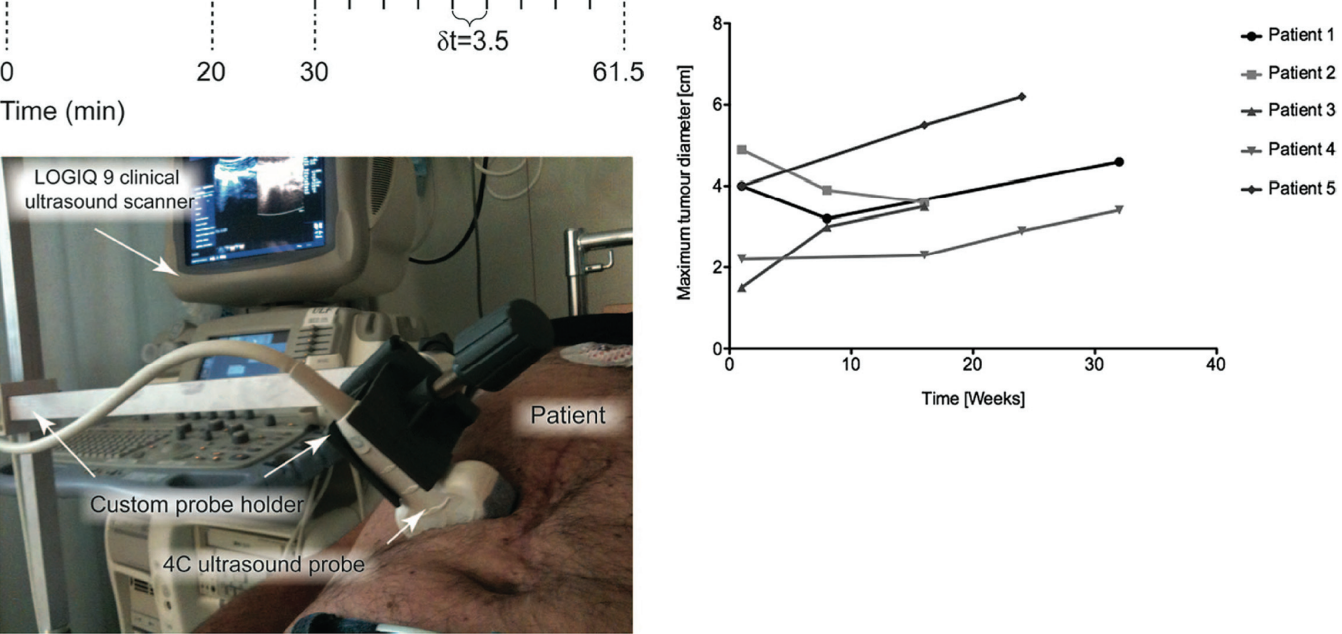

FIGURE 7| Treatment of human pancreatic adenocarcinoma using gemcitabine using microbubble-assisted ultrasound (Adapted with permission from Kotopoulis et al., 2013 - Copyright (C) 2013 Am. Assoc. Phys. Med.). (A) Timeframe of each sonochemotherapy treatment schedule, arrows indicate intravenous injection of $0.5 \mathrm{ml}$

SonoVue followed by a 5-ml injection of saline, $\delta$ t represents the time between each injection; (B) Photo of the probe and custom-made probe holder during patient treatment; (C) Changes in tumor diameter over time measured from CT images in patients with pancreatic malignancy. 
in $20 \mathrm{~min}$. In addition to the safety of the therapeutic protocol, the authors will explore the largest MI and ultrasound treatment time patients can tolerate. The secondary objectives of this clinical trial are to observe the tumor clinical benefit rate and to evaluate the preliminary effects including time to failure and time to death.

\section{Conclusion}

Targeted drug delivery using microbubble-assisted ultrasound has the potential to become a clinically accepted way of improving local anticancer chemotherapy. Although the coadministration approach, using clinically approved microbubbles and free chemotherapeutic drugs, can be seen as the fasttrack toward the clinic, the greatest therapeutic potential may lie in the custom-made drug-loaded microbubbles. The latter combines the enhanced vascular permeability and cellular uptake following microbubble-assisted ultrasound with a local release of the drug. However, this implies that new therapeutic particles are to be developed, which require thorough

\section{References}

Al Sabbagh, C., Tsapis, N., Novell, A., Calleja-Gonzalez, P., Escoffre, J. M., Bouakaz, A., et al. (2015). Formulation and pharmacokinetics of thermosensitive stealth(R) liposomes encapsulating 5-Fluorouracil. Pharm. Res. 32, 1585-1603. doi: 10.1007/s11095-014-1559-0

Aryal, M., Arvanitis, C. D., Alexander, P. M., and Mcdannold, N. (2014). Ultrasound-mediated blood-brain barrier disruption for targeted drug delivery in the central nervous system. Adv. Drug Deliv. Rev. 72, 94-109. doi: 10.1016/j.addr.2014.01.008

Azagury, A., Khoury, L., Enden, G., and Kost, J. (2014). Ultrasound mediated transdermal drug delivery. Adv. Drug Deliv. Rev. 72, 127-143. doi: 10.1016/j.addr.2014.01.007

Bohmer, M. R., Chlon, C. H., Raju, B. I., Chin, C. T., Shevchenko, T., and Klibanov, A. L. (2010). Focused ultrasound and microbubbles for enhanced extravasation. J. Control. Release 148, 18-24. doi: 10.1016/j.jconrel.2010.06.012

Bokor, D., Chambers, J. B., Rees, P. J., Mant, T. G., Luzzani, F., and Spinazzi, A. (2001). Clinical safety of SonoVue, a new contrast agent for ultrasound imaging, in healthy volunteers and in patients with chronic obstructive pulmonary disease. Invest. Radiol. 36, 104-109. doi: 10.1097/00004424-200102000-00006

Boucher, Y., Baxter, L. T., and Jain, R. K. (1990). Interstitial pressure gradients in tissue-isolated and subcutaneous tumors: implications for therapy. Cancer Res. 50, 4478-4484

Burke, C. W., Alexander, E. T., Timbie, K., Kilbanov, A. L., and Price, R. J. (2014). Ultrasound-activated agents comprised of 5FU-bearing nanoparticles bonded to microbubbles inhibit solid tumor growth and improve survival. Mol. Ther 22, 321-328. doi: 10.1038/mt.2013.259

Burke, C. W., Klibanov, A. L., Sheehan, J. P., and Price, R. J. (2011). Inhibition of glioma growth by microbubble activation in a subcutaneous model using low duty cycle ultrasound without significant heating. J. Neurosurg. 114, 1654-1661. doi: 10.3171/2010.11.JNS101201

Cavalieri, F., Zhou, M., and Ashokkumar, M. (2010). The design of multifunctional microbubbles for ultrasound image-guided cancer therapy. Curr. Top. Med Chem. 10, 1198-1210. doi: 10.2174/156802610791384180

Chauhan, V. P., Stylianopoulos, T., Boucher, Y., and Jain, R. K. (2011). Delivery of molecular and nanoscale medicine to tumors: transport barriers and strategies. Annu. Rev. Chem. Biomol. Eng. 2, 281-298. doi: 10.1146/annurev-chembioeng061010-114300

Choi, J. J., Pernot, M., Brown, T. R., Small, S. A., and Konofagou, E. E. (2007) Spatio-temporal analysis of molecular delivery through the blood-brain barrier using focused ultrasound. Phys. Med. Biol. 52, 5509-5530. doi: 10.1088/0031$9155 / 52 / 18 / 004$ pre-clinical testing for efficacy and safety. A growing number of preclinical experiments have successfully reported the therapeutic benefits of microbubble-assisted ultrasound in the delivery of (anti-cancer) drugs in several animal models. Clinical translation of this method requires further improvements on: (i) the design, characterization, and GMP production of therapeutic microbubbles with prolonged plasma half-life and high drug-loading capacity; (ii) the optimization and standardization of ultrasound parameters used in the field; (iii) the insertion of a medical imaging modality (MRI, ultrasound) to monitor the in vivo effects of ultrasound and (iv) the evaluation of drug biodistribution, therapeutic efficacy, and side effects in orthotopic tumor models in small and large animals.

\section{Acknowledgment}

This work was supported by Advanced ERC grant Sound Pharma - 268906 (CM).

Cochran, M. C., Eisenbrey, J., Ouma, R. O., Soulen, M., and Wheatley, M. A. (2011). Doxorubicin and paclitaxel loaded microbubbles for ultrasound triggered drug delivery. Int. J. Pharm. 414, 161-170. doi: 10.1016/j.ijpharm.2011.05.030

De Cock, I., Zagato, E., Braeckmans, K., Luan, Y., de Jong, N., De Smedt, S. C., et al. (2015). Ultrasound and microbubble mediated drug delivery: acoustic pressure as determinant for uptake via membrane pores or endocytosis. J. Control. Release 197, 20-28. doi:10.1016/j.jconrel.2014.10.031

Deckers, R., and Moonen, C. T. (2010). Ultrasound triggered, image guided, local drug delivery. J. Control. Release 148, 25-33. doi: 10.1016/j.jconrel.2010.07.117

Deng, J., Huang, Q., Wang, F., Liu, Y., Wang, Z., Wang, Z., et al. (2012). The role of caveolin-1 in blood-brain barrier disruption induced by focused ultrasound combined with microbubbles. J. Mol. Neurosci. 46, 677-687. doi 10.1007/s12031-011-9629-9

Deng, Z., Yan, F., Jin, Q., Li, F., Wu, J., Liu, X., et al. (2014). Reversal of multidrug resistance phenotype in human breast cancer cells using doxorubicin-liposomemicrobubble complexes assisted by ultrasound. J. Control. Release 174, 109-116. doi: 10.1016/j.jconrel.2013.11.018

Deshpande, N., Needles, A., and Willmann, J. K. (2010). Molecular ultrasound imaging: current status and future directions. Clin. Radiol. 65, 567-581. doi: 10.1016/j.crad.2010.02.013

Doinikov, A. A., and Bouakaz, A. (2010). Acoustic microstreaming around an encapsulated particle. J. Acoust. Soc. Am. 127, 1218-1227. doi: $10.1121 / 1.3290997$

Escoffre, J. M., Mannaris, C., Geers, B., Novell, A., Lentacker, I., Averkiou, M et al. (2013a). Doxorubicin liposome-loaded microbubbles for contrast imaging and ultrasound-triggered drug delivery. IEEE Trans. Ultrason. Ferroelectr. Freq. Control 60, 78-87. doi: 10.1109/TUFFC.2013.2539

Escoffre, J. M., Novell, A., Serriere, S., Lecomte, T., and Bouakaz, A. (2013b). Irinotecan delivery by microbubble-assisted ultrasound: in vitro validation and a pilot preclinical study. Mol. Pharm. 10, 2667-2675. doi: 10.1021/mp400081b

Escoffre, J. M., Zeghimi, A., Novell, A., and Bouakaz, A. (2013c). In-vivo gene delivery by sonoporation: recent progress and prospects. Curr. Gene Ther. 13, 2-14. doi: 10.2174/156652313804806606

Escoffre, J. M., Piron, J., Novell, A., and Bouakaz, A. (2011). Doxorubicin delivery into tumor cells with ultrasound and microbubbles. Mol. Pharm. 8, 799-806. doi: $10.1021 / \mathrm{mp} 100397 \mathrm{p}$

Fan, C. H., Ting, C. Y., Liu, H. L., Huang, C. Y., Hsieh, H. Y., Yen, T. C., et al. (2013). Antiangiogenic-targeting drug-loaded microbubbles combined with focused ultrasound for glioma treatment. Biomaterials 34, 2142-2155. doi: 10.1016/j.biomaterials.2012.11.048

Ferlay, J., Steliarova-Foucher, E., Lortet-Tieulent, J., Rosso, S., Coebergh, J. W., Comber, H., et al. (2013). Cancer incidence and mortality patterns in Europe: 
estimates for 40 countries in 2012. Eur. J. Cancer 49, 1374-1403. doi: 10.1016/j.ejca.2012.12.027

Fokong, S., Theek, B., Wu, Z., Koczera, P., Appold, L., Jorge, S., et al. (2012). Imageguided, targeted and triggered drug delivery to tumors using polymer-based microbubbles. J. Control. Release 163, 75-81. doi: 10.1016/j.jconrel.2012.05.007

Frenkel, V. (2008). Ultrasound mediated delivery of drugs and genes to solid tumors. Adv. Drug Deliv. Rev. 60, 1193-1208. doi: 10.1016/j.addr.2008.03.007

Frinking, P. J., Tardy, I., Theraulaz, M., Arditi, M., Powers, J., Pochon, S., et al. (2012). Effects of acoustic radiation force on the binding efficiency of BR55, a VEGFR2-specific ultrasound contrast agent. Ultrasound Med. Biol. 38, 14601469. doi: 10.1016/j.ultrasmedbio.2012.03.018

Gao, Y., Gao, S., Zhao, B., Zhao, Y., Hua, X., Tan, K., et al. (2012). Vascular effects of microbubble-enhanced, pulsed, focused ultrasound on liver blood perfusion. Ultrasound Med. Biol. 38, 91-98. doi: 10.1016/j.ultrasmedbio.2011.09.018

Geers, B., Lentacker, I., Sanders, N. N., Demeester, J., Meairs, S., and De Smedt, S. C. (2011). Self-assembled liposome-loaded microbubbles: the missing link for safe and efficient ultrasound triggered drug-delivery. J. Control. Release 152, 249-256. doi: 10.1016/j.jconrel.2011.02.024

Goertz, D. E. (2015). An overview of the influence of therapeutic ultrasound exposures on the vasculature: high intensity ultrasound and microbubble-mediated bioeffects. Int. J. Hyperthermia 31, 134-144. doi: 10.3109/02656736.2015.1009179

Grauer, S. E., Sutherland, G., and Fritz, T. (1996). "Safety and echo contrast efficacy of multiple doses of Aerosomes MRX-115 in a phase I clinical trial," in Proceedings of the AHA, 69th Scientific Sessions, Circulation, New Orleans, LA, I316-I319.

Hancock, H. A., Smith, L. H., Cuesta, J., Durrani, A. K., Angstadt, M., Palmeri, M. L., et al. (2009). Investigations into pulsed high-intensity focused ultrasound-enhanced delivery: preliminary evidence for a novel mechanism. Ultrasound Med. Biol. 35, 1722-1736. doi: 10.1016/j.ultrasmedbio.2009.04.020

Heath, C. H., Sorace, A., Knowles, J., Rosenthal, E., and Hoyt, K. (2012). Microbubble therapy enhances anti-tumor properties of cisplatin and cetuximab in vitro and in vivo. Otolaryngol. Head Neck Surg. 146, 938-945. doi: $10.1177 / 0194599812436648$

Hernot, S., and Klibanov, A. L. (2008). Microbubbles in ultrasound-triggered drug and gene delivery. Adv. Drug Deliv. Rev. 60, 1153-1166. doi: 10.1016/j.addr.2008.03.005

Hijnen, N., Langereis, S., and Grull, H. (2014). Magnetic resonance guided high-intensity focused ultrasound for image-guided temperature-induced drug delivery. Adv. Drug Deliv. Rev. 72, 65-81. doi: 10.1016/j.addr.2014.01.006

$\mathrm{Hu}, \mathrm{X}$. , Kheirolomoom, A., Mahakian, L. M., Beegle, J. R., Kruse, D. E., Lam, K. S., et al. (2012). Insonation of targeted microbubbles produces regions of reduced blood flow within tumor vasculature. Invest. Radiol. 47, 398-405. doi: 10.1097/RLI.0b013e31824bd237

Ibsen, S., Schutt, C. E., and Esener, S. (2013). Microbubble-mediated ultrasound therapy: a review of its potential in cancer treatment. Drug Des. Devel. Ther. 7 , 375-388. doi: 10.2147/DDDT.S31564

Iwanaga, K., Tominaga, K., Yamamoto, K., Habu, M., Maeda, H., Akifusa, S., et al. (2007). Local delivery system of cytotoxic agents to tumors by focused sonoporation. Cancer Gene Ther. 14, 354-363. doi: 10.1038/sj.cgt.77 01026

Jain, R. K. (2001). Delivery of molecular and cellular medicine to solid tumors. $A d v$. Drug Deliv. Rev. 46, 149-168. doi: 10.1016/S0169-409X(00)00131-9

Juffermans, L. J., Kamp, O., Dijkmans, P. A., Visser, C. A., and Musters, R. J. (2008). Low-intensity ultrasound-exposed microbubbles provoke local hyperpolarization of the cell membrane via activation of $\mathrm{BK}(\mathrm{Ca})$ channels. Ultrasound Med. Biol. 34, 502-508. doi: 10.1016/j.ultrasmedbio.2007.09.010

Juffermans, L. J., Van Dijk, A., Jongenelen, C. A., Drukarch, B., Reijerkerk, A., De Vries, H. E., et al. (2009). Ultrasound and microbubble-induced intra- and intercellular bioeffects in primary endothelial cells. Ultrasound Med. Biol. 35, 1917-1927. doi: 10.1016/j.ultrasmedbio.2009.06.1091

Junge, L., Ohl, C. D., Wolfrum, B., Arora, M., and Ikink, R. (2003). Cell detachment method using shock-wave-induced cavitation. Ultrasound Med. Biol. 29, 17691776. doi: 10.1016/j.ultrasmedbio.2003.08.010

Kaddur, K., Lebegue, L., Tranquart, F., Midoux, P., Pichon, C., and Bouakaz, A. (2010). Transient transmembrane release of green fluorescent proteins with sonoporation. IEEE Trans. Ultrason. Ferroelectr. Freq. Control 57, 1558-1567. doi: 10.1109/TUFFC.2010.1586
Kang, J., Wu, X., Wang, Z., Ran, H., Xu, C., Wu, J., et al. (2010). Antitumor effect of docetaxel-loaded lipid microbubbles combined with ultrasound-targeted microbubble activation on VX2 rabbit liver tumors. J. Ultrasound Med. 29, $61-70$.

Kiessling, F., Fokong, S., Bzyl, J., Lederle, W., Palmowski, M., and Lammers, T. (2014). Recent advances in molecular, multimodal and theranostic ultrasound imaging. Adv. Drug Deliv. Rev. 72, 15-27. doi: 10.1016/j.addr.2013.11.013

Kiessling, F., Fokong, S., Koczera, P., Lederle, W., and Lammers, T. (2012). Ultrasound microbubbles for molecular diagnosis, therapy, and theranostics. J. Nucl. Med. 53, 345-348. doi: 10.2967/jnumed.111.099754

Kinoshita, M., Mcdannold, N., Jolesz, F. A., and Hynynen, K. (2006). Noninvasive localized delivery of Herceptin to the mouse brain by MRI-guided focused ultrasound-induced blood-brain barrier disruption. Proc. Natl. Acad. Sci. U.S.A. 103, 11719-11723. doi: 10.1073/pnas.0604318103

Kong, G., Braun, R. D., and Dewhirst, M. W. (2001). Characterization of the effect of hyperthermia on nanoparticle extravasation from tumor vasculature. Cancer Res. 61, 3027-3032.

Kooiman, K., Emmer, M., Foppen-Harteveld, M., Van Wamel, A., and De Jong, N. (2010). Increasing the endothelial layer permeability through ultrasound-activated microbubbles. IEEE Trans. Biomed. Eng. 57, 29-32. doi: 10.1109/TBME.2009.2030335

Kooiman, K., Vos, H. J., Versluis, M., and De Jong, N. (2014). Acoustic behavior of microbubbles and implications for drug delivery. Adv. Drug Deliv. Rev. 72, 28-48. doi: 10.1016/j.addr.2014.03.003

Kotopoulis, S., Delalande, A., Popa, M., Mamaeva, V., Dimcevski, G., Gilja, O. H., et al. (2014). Sonoporation-enhanced chemotherapy significantly reduces primary tumour burden in an orthotopic pancreatic cancer xenograft. Mol. Imaging Biol. 16, 53-62. doi: 10.1007/s11307-013-0672-5

Kotopoulis, S., Dimcevski, G., Gilja, O. H., Hoem, D., and Postema, M. (2013). Treatment of human pancreatic cancer using combined ultrasound, microbubbles, and gemcitabine: a clinical case study. Med. Phys. 40, 072902. doi: $10.1118 / 1.4808149$

Kotopoulis, S., Dimcevski, G., Hoem, D., Postema, M., and Gilja, O. H. (2015). "Therapeutic ultrasound in pancreatic adenocarcinoma - Oral communication," in Proceedings of the 20th European Symposium on Ultrasound Contrast Imaging, Rotterdam.

Lammers, T., Kiessling, F., Hennink, W. E., and Storm, G. (2012). Drug targeting to tumors: principles, pitfalls and (pre-) clinical progress. J. Control. Release 161, 175-187. doi: 10.1016/j.jconrel.2011.09.063

Lammers, T., Koczera, P., Fokong, S., Gremse, F., Ehling, J., Vogt, M., et al. (2015). Theranostic USPIO-loaded microbubbles for mediating and monitoring blood-brain barrier permeation. Adv. Funct. Mater. 25, 36-43. doi: 10.1002/adfm.201401199

Lammertink, B., Deckers, R., Storm, G., Moonen, C., and Bos, C. (2015). Duration of ultrasound-mediated enhanced plasma membrane permeability. Int. J. Pharm. 482, 92-98. doi: 10.1016/j.ijpharm.2014.12.013

Lefor, A. T., Makohon, S., and Ackerman, N. B. (1985). The effects of hyperthermia on vascular permeability in experimental liver metastasis. J. Surg. Oncol. 28, 297-300. doi: 10.1002/jso.2930280412

Leighton, T. G. (1994). The Acoustic Bubble. London: Elsevier.

Lentacker, I., De Cock, I., Deckers, R., De Smedt, S. C., and Moonen, C. T. (2014). Understanding ultrasound induced sonoporation: definitions and underlying mechanisms. Adv. Drug Deliv. Rev. 72, 49-64. doi: 10.1016/j.addr.2013.11.008

Li, P., Zheng, Y., Ran, H., Tan, J., Lin, Y., Zhang, Q., et al. (2012). Ultrasound triggered drug release from 10-hydroxycamptothecin-loaded phospholipid microbubbles for targeted tumor therapy in mice. J. Control. Release 162, 349-354. doi: 10.1016/j.jconrel.2012.07.009

Lin, C. Y., Tseng, H. C., Shiu, H. R., Wu, M. F., Chou, C. Y., and Lin, W. L. (2012). Ultrasound sonication with microbubbles disrupts blood vessels and enhances tumor treatments of anticancer nanodrug. Int. J. Nanomedicine 7, 2143-2152. doi: 10.2147/IJN.S29514

Lindner, L. H., Eichhorn, M. E., Eibl, H., Teichert, N., Schmitt-Sody, M., Issels, R. D., et al. (2004). Novel temperature-sensitive liposomes with prolonged circulation time. Clin. Cancer Res. 10, 2168-2178. doi: 10.1158/1078-0432.CCR03-0035

Lu, C. T., Zhao, Y. Z., Wu, Y., Tian, X. Q., Li, W. F., Huang, P. T., et al. (2011). Experiment on enhancing antitumor effect of intravenous epirubicin hydrochloride by acoustic cavitation in situ combined with 
phospholipid-based microbubbles. Cancer Chemother. Pharmacol. 68, 343-348. doi: 10.1007/s00280-010-1489-4

Lum, A. F., Borden, M. A., Dayton, P. A., Kruse, D. E., Simon, S. I., and Ferrara, K. W. (2006). Ultrasound radiation force enables targeted deposition of model drug carriers loaded on microbubbles. J. Control. Release 111, 128-134. doi: 10.1016/j.jconrel.2005.11.006

Manzoor, A. A., Lindner, L. H., Landon, C. D., Park, J. Y., Simnick, A. J., Dreher, M. R., et al. (2012). Overcoming limitations in nanoparticle drug delivery: triggered, intravascular release to improve drug penetration into tumors. Cancer Res. 72, 5566-5575. doi: 10.1158/0008-5472.CAN-12-1683

Marty, B., Larrat, B., Van Landeghem, M., Robic, C., Robert, P., Port, M., et al. (2012). Dynamic study of blood-brain barrier closure after its disruption using ultrasound: a quantitative analysis. J. Cereb. Blood Flow Metab. 32, 1948-1958. doi: $10.1038 /$ jcbfm. 2012.100

Matsuo, M., Yamaguchi, K., Feril, L. B. Jr., Endo, H., Ogawa, K., Tachibana, K., et al. (2011). Synergistic inhibition of malignant melanoma proliferation by melphalan combined with ultrasound and microbubbles. Ultrason. Sonochem. 18, 1218-1224. doi: 10.1016/j.ultsonch.2011.03.005

Mei, J., Cheng, Y., Song, Y., Yang, Y., Wang, F., Liu, Y., et al. (2009). Experimental study on targeted methotrexate delivery to the rabbit brain via magnetic resonance imaging-guided focused ultrasound. J. Ultrasound Med. 28, 871-880.

Meijering, B. D., Juffermans, L. J., Van Wamel, A., Henning, R. H., Zuhorn, I. S., Emmer, M., et al. (2009). Ultrasound and microbubble-targeted delivery of macromolecules is regulated by induction of endocytosis and pore formation. Circ. Res. 104, 679-687. doi: 10.1161/CIRCRESAHA.108. 183806

Mesiwala, A. H., Farrell, L., Wenzel, H. J., Silbergeld, D. L., Crum, L. A., Winn, H. R., et al. (2002). High-intensity focused ultrasound selectively disrupts the blood-brain barrier in vivo. Ultrasound Med. Biol. 28, 389-400. doi: 10.1016/S0301-5629(01)00521-X

Minchinton, A. I., and Tannock, I. F. (2006). Drug penetration in solid tumours. Nat. Rev. Cancer 6, 583-592. doi: 10.1038/nrc1893

Minnaert, M. (1933). On musical air-bubbles and the sounds of running water. Philos. Mag. 16, 235-249. doi: 10.1080/14786443309462277

Novell, A., Escoffre, J. M., and Bouakaz, A. (2013). Ultrasound contrast imaging in Cancer - Technical aspects and prospects. Curr. Mol. Imaging 2, 77-88. doi: 10.2174/2211555211302010009

Ohl, C. D., Arora, M., Ikink, R., De Jong, N., Versluis, M., Delius, M., et al. (2006). Sonoporation from jetting cavitation bubbles. Biophys. J. 91, 4285-4295. doi: 10.1529/biophysj.105.075366

Ohl, C. D., and Wolfrum, B. (2003). Detachment and sonoporation of adherent HeLa-cells by shock wave-induced cavitation. Biochim. Biophys. Acta 1624, 131-138. doi: 10.1016/j.bbagen.2003.10.005

Podaru, G., Ogden, S., Baxter, A., Shrestha, T., Ren, S., Thapa, P., et al. (2014). Pulsed magnetic field induced fast drug release from magneto liposomes via ultrasound generation. J. Phys. Chem. B 118, 11715-11722. doi: $10.1021 /$ jp5022278

Postema, M., Van Wamel, A., Ten Cate, F. J., and De Jong, N. (2005). High-speed photography during ultrasound illustrates potential therapeutic applications of microbubbles. Med. Phys. 32, 3707-3711. doi: 10.1118/1.21 33718

Price, R. J., Skyba, D. M., Kaul, S., and Skalak, T. C. (1998). Delivery of colloidal particles and red blood cells to tissue through microvessel ruptures created by targeted microbubble destruction with ultrasound. Circulation 98, 1264-1267. doi: 10.1161/01.CIR.98.13.1264

Pron, G., Mahrour, N., Orlowski, S., Tounekti, O., Poddevin, B., Belehradek, J., et al. (1999). Internalisation of the bleomycin molecules responsible for bleomycin toxicity: a receptor-mediated endocytosis mechanism. Biochem. Pharmacol. 57, 45-56. doi: 10.1016/S0006-2952(98)00282-2

$\mathrm{Pu}$, C., Chang, S., Sun, J., Zhu, S., Liu, H., Zhu, Y., et al. (2014). Ultrasound-mediated destruction of LHRHa-targeted and paclitaxel-loaded lipid microbubbles for the treatment of intraperitoneal ovarian cancer xenografts. Mol. Pharm. 11, 49-58. doi: 10.1021/mp400523h

Qin, J., Wang, T. Y., and Willman, J. K. (2015). "Sonoporation: applications for cancer therapy," in Therapeutic Ultrasound, eds J. M. Escoffre and A. Bouakaz (Berlin: Springer).
Raymond, S. B., Skoch, J., Hynynen, K., and Bacskai, B. J. (2007). Multiphoton imaging of ultrasound/Optison mediated cerebrovascular effects in vivo. J. Cereb. Blood Flow Metab. 27, 393-403. doi: 10.1038/sj.jcbfm.9600336

Ren, S. T., Liao, Y. R., Kang, X. N., Li, Y. P., Zhang, H., Ai, H., et al. (2013). The antitumor effect of a new docetaxel-loaded microbubble combined with lowfrequency ultrasound in vitro: preparation and parameter analysis. Pharm. Res. 30, 1574-1585. doi: 10.1007/s11095-013-0996-5

Rychak, J. J., and Klibanov, A. L. (2014). Nucleic acid delivery with microbubbles and ultrasound. Adv. Drug Deliv. Rev. 72, 82-93. doi: 10.1016/j.addr.2014.01.009

Rychak, J. J., Klibanov, A. L., and Hossack, J. A. (2005). Acoustic radiation force enhances targeted delivery of ultrasound contrast microbubbles: in vitro verification. IEEE Trans. Ultrason. Ferroelectr. Freq. Control 52, 421-433. doi: 10.1109/TUFFC.2005.1417264

Sanches, P. G., Grull, H., and Steinbach, O. C. (2011). See, reach, treat: ultrasound-triggered image-guided drug delivery. Ther. Deliv. 2, 919-934. doi: $10.4155 /$ tde. 11.63

Sarvazyan, A. P., Rudenko, O. V., and Nyborg, W. L. (2010). Biomedical applications of radiation force of ultrasound: historical roots and physical basis. Ultrasound Med. Biol. 36, 1379-1394. doi: 10.1016/j.ultrasmedbio.2010.05.015

Sasaki, N., Kudo, N., Nakamura, K., Lim, S., et al. (2014). Ultrasound image-guided therapy enhances antitumor effect of cisplatin. J. Med. Ultrasonics 41, 11-21. doi: $10.1007 /$ s10396-013-0475-y

Seidl, M., Steinbach, P., Worle, K., and Hofstadter, F. (1994). Induction of stress fibres and intercellular gaps in human vascular endothelium by shock-waves. Ultrasonics 32, 397-400. doi: 10.1016/0041-624X(94)90111-2

Sersa, G., Miklavcic, D., Cemazar, M., Rudolf, Z., Pucihar, G., and Snoj, M. (2008). Electrochemotherapy in treatment of tumours. Eur. J. Surg. Oncol. 34, 232-240. doi: 10.1016/j.ejso.2007.05.016

Sheikov, N., Mcdannold, N., Sharma, S., and Hynynen, K. (2008). Effect of focused ultrasound applied with an ultrasound contrast agent on the tight junctional integrity of the brain microvascular endothelium. Ultrasound Med. Biol. 34, 1093-1104. doi: 10.1016/j.ultrasmedbio.2007.12.015

Sheikov, N., Mcdannold, N., Vykhodtseva, N., Jolesz, F., and Hynynen, K. (2004). Cellular mechanisms of the blood-brain barrier opening induced by ultrasound in presence of microbubbles. Ultrasound Med. Biol. 30, 979-989. doi: 10.1016/j.ultrasmedbio.2004.04.010

Shortencarier, M. J., Dayton, P. A., Bloch, S. H., Schumann, P. A., Matsunaga, T. O., and Ferrara, K. W. (2004). A method for radiation-force localized drug delivery using gas-filled lipospheres. IEEE Trans. Ultrason. Ferroelectr. Freq. Control 51, 822-831. doi: 10.1109/TUFFC.2004.1320741

Sirsi, S. R., and Borden, M. A. (2014). State-of-the-art materials for ultrasound-triggered drug delivery. Adv. Drug Deliv. Rev. 72, 3-14. doi: 10.1016/j.addr.2013.12.010

Sklar, L. R., Burnett, C. T., Waibel, J. S., Moy, R. L., and Ozog, D. M. (2014). Laser assisted drug delivery: a review of an evolving technology. Lasers Surg. Med. 46, 249-262. doi: 10.1002/lsm.22227

Song, C. W. (1984). Effect of local hyperthermia on blood flow and microenvironment: a review. Cancer Res. 44, 4721s-4730s.

Song, J., Chappell, J. C., Qi, M., Vangieson, E. J., Kaul, S., and Price, R. J. (2002). Influence of injection site, microvascular pressure and ultrasound variables on microbubble-mediated delivery of microspheres to muscle. J. Am. Coll. Cardiol. 39, 726-731. doi: 10.1016/S0735-1097(01)01793-4

Sonoda, S., Tachibana, K., Uchino, E., Yamashita, T., Sakoda, K., Sonoda, K. H., et al. (2007). Inhibition of melanoma by ultrasound-microbubble-aided drug delivery suggests membrane permeabilization. Cancer Biol. Ther. 6, 1276-1283. doi: $10.4161 /$ cbt.6.8.4485

Sorace, A. G., Warram, J. M., Umphrey, H., and Hoyt, K. (2012). Microbubblemediated ultrasonic techniques for improved chemotherapeutic delivery in cancer. J. Drug Target. 20, 43-54. doi: 10.3109/1061186X.2011. 622397

Stieger, S. M., Caskey, C. F., Adamson, R. H., Qin, S., Curry, F. R., Wisner, E. R., et al. (2007). Enhancement of vascular permeability with lowfrequency contrast-enhanced ultrasound in the chorioallantoic membrane model. Radiology 243, 112-121. doi: 10.1148/radiol.2431060167

Szabo, T. L. (2013). Diagnostic Ultrasound Imaging: Inside Out. Waltham, MA: Academic Press. 
ter Haar, G., Shaw, A., Pye, S., Ward, B., Bottomley, F., Nolan, R., et al. (2011). Guidance on reporting ultrasound exposure conditions for bio-effects studies. Ultrasound Med. Biol. 37, 177-183. doi: 10.1016/j.ultrasmedbio.2010.10.021

Ting, C. Y., Fan, C. H., Liu, H. L., Huang, C. Y., Hsieh, H. Y., Yen, T. C., et al. (2012). Concurrent blood-brain barrier opening and local drug delivery using drugcarrying microbubbles and focused ultrasound for brain glioma treatment. Biomaterials 33, 704-712. doi: 10.1016/j.biomaterials.2011.09.096

Tinkov, S., Coester, C., Serba, S., Geis, N. A., Katus, H. A., Winter, G., et al. (2010). New doxorubicin-loaded phospholipid microbubbles for targeted tumor therapy: in-vivo characterization. J. Control. Release 148, 368-372. doi: 10.1016/j.jconrel.2010.09.004

Todorova, M., Agache, V., Mortazavi, O., Chen, B., Karshafian, R., Hynynen, K., et al. (2013). Antitumor effects of combining metronomic chemotherapy with the antivascular action of ultrasound stimulated microbubbles. Int. J. Cancer 132, 2956-2966. doi: 10.1002/ijc.27977

Tran, T. A., Roger, S., Le Guennec, J. Y., Tranquart, F., and Bouakaz, A. (2007). Effect of ultrasound-activated microbubbles on the cell electrophysiological properties. Ultrasound Med. Biol. 33, 158-163. doi: 10.1016/j.ultrasmedbio.2006.07.029

Treat, L. H., Mcdannold, N., Zhang, Y., Vykhodtseva, N., and Hynynen, K. (2012). Improved anti-tumor effect of liposomal doxorubicin after targeted blood-brain barrier disruption by MRI-guided focused ultrasound in rat glioma. Ultrasound Med. Biol. 38, 1716-1725. doi: 10.1016/j.ultrasmedbio.2012. 04.015

Tzu-Yin, W., Wilson, K. E., Machtaler, S., and Willman, J. K. (2014). Ultrasound and microbubble guided drug delivery: mechanistic understanding and clinical implications. Curr. Pharm. Biotechnol. 14, 743-752. doi: 10.2174/1389201014666131226114611

Unga, J., and Hashida, M. (2014). Ultrasound induced cancer immunotherapy. Adv. Drug Deliv. Rev. 72, 144-153. doi: 10.1016/j.addr.2014.03.004

Unger, E., Porter, T., Lindner, J., and Grayburn, P. (2014). Cardiovascular drug delivery with ultrasound and microbubbles. Adv. Drug Deliv. Rev. 72, 110-126. doi: 10.1016/j.addr.2014.01.012

van Wamel, A., Kooiman, K., Harteveld, M., Emmer, M., Ten Cate, F. J., Versluis, M., et al. (2006). Vibrating microbubbles poking individual cells: drug transfer into cells via sonoporation. J. Control. Release 112, 149-155. doi: 10.1016/j.jconrel.2006.02.007

Vaupel, P. W., and Kelleher, D. K. (2012). Blood flow and associated pathophysiology of uterine cervix cancers: characterisation and relevance for localised hyperthermia. Int. J. Hyperthermia 28, 518-527. doi: $10.3109 / 02656736.2012 .699134$
Wang, S., Hossack, J. A., Klibanov, A. L., and Mauldin, F. W. Jr. (2014). Binding dynamics of targeted microbubbles in response to modulated acoustic radiation force. Phys. Med. Biol. 59, 465-484. doi: 10.1088/0031-9155/59/2/465

Wei, K. C., Chu, P. C., Wang, H. Y., Huang, C. Y., Chen, P. Y., Tsai, H. C., et al. (2013). Focused ultrasound-induced blood-brain barrier opening to enhance temozolomide delivery for glioblastoma treatment: a preclinical study. PLoS ONE 8:e58995. doi: 10.1371/journal.pone.0058995

Weinberg, R. A. (2014). The Biology of Cancer. New York, NY: Garland Science.

Wilson, S. R., and Burns, P. N. (2010). Microbubble-enhanced US in body imaging: what role? Radiology 257, 24-39. doi: 10.1148/radiol.10091210

$\mathrm{Wu}$, J. (2002). Theoretical study on shear stress generated by microstreaming surrounding contrast agents attached to living cells. Ultrasound Med. Biol. 28, 125-129. doi: 10.1016/S0301-5629(01)00497-5

Yan, F., Li, L., Deng, Z., Jin, Q., Chen, J., Yang, W., et al. (2013). Paclitaxel-liposomemicrobubble complexes as ultrasound-triggered therapeutic drug delivery carriers. J. Control. Release 166, 246-255. doi: 10.1016/j.jconrel.2012.12.025

Yan, K., and Shen, L. (2014). Safety Study of Combining Ultrasound Microbubbles and Chemotherapy to Treat Malignant Neoplasms of Digestive System. Available at: https://clinicaltrials.gov/ct2/show/study/NCT02233205

Yang, F., Gu, N., Chen, D., Xi, X., Zhang, D., Li, Y., et al. (2008). Experimental study on cell self-sealing during sonoporation. J. Control. Release 131, 205-210. doi: 10.1016/j.jconrel.2008.07.038

Yatvin, M. B., Weinstein, J. N., Dennis, W. H., and Blumenthal, R. (1978). Design of liposomes for enhanced local release of drugs by hyperthermia. Science 202, 1290-1293. doi: 10.1126/science. 364652

Zhao, Y. Z., Lu, C. T., Zhou, Z. C., Jin, Z., Zhang, L., Sun, C. Z., et al. (2011). Enhancing chemotherapeutic drug inhibition on tumor growth by ultrasound: an in vivo experiment. J. Drug Target. 19, 154-160. doi: $10.3109 / 10611861003801834$

Conflict of Interest Statement: The authors declare that the research was conducted in the absence of any commercial or financial relationships that could be construed as a potential conflict of interest.

Copyright (c) 2015 Lammertink, Bos, Deckers, Storm, Moonen and Escoffre. This is an open-access article distributed under the terms of the Creative Commons Attribution License (CC BY). The use, distribution or reproduction in other forums is permitted, provided the original author(s) or licensor are credited and that the original publication in this journal is cited, in accordance with accepted academic practice. No use, distribution or reproduction is permitted which does not comply with these terms. 